\title{
CLASH: Photometric redshifts with 16 HST bands in galaxy cluster fields ${ }^{\star, \star \star}$
}

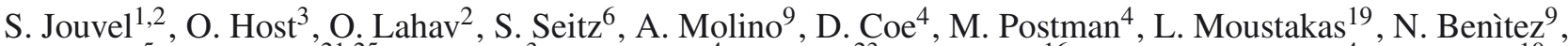 \\ P. Rosati ${ }^{5}$, I. Balestra ${ }^{21,25}$, C. Grillo ${ }^{3}$, L. Bradley ${ }^{4}$, A. Fritz ${ }^{23}$, D. Kelson ${ }^{16}$, A. M. Koekemoer ${ }^{4}$, D. Lemze ${ }^{10}$, \\ E. Medezinski ${ }^{10}$, A. Mercurio ${ }^{21}$, J. Moustakas ${ }^{22}$, M. Nonino ${ }^{25}$, M. Scodeggio ${ }^{23}$, W. Zheng ${ }^{10}$, A. Zitrin ${ }^{24,26}$, \\ M. Bartelmann ${ }^{11}$, R. Bouwens ${ }^{12}$, T. Broadhurst ${ }^{7,8}$, M. Donahue ${ }^{13}$, H. Ford ${ }^{10}$, G. Graves $^{14}$, L. Infante $^{15}$, \\ Y. Jimenez-Teja ${ }^{9}$, R. Lazkoz ${ }^{7}$, P. Melchior ${ }^{17}$, M. Meneghetti ${ }^{18}$, J. Merten ${ }^{19}$, S. Ogaz ${ }^{4}$, and K. Umetsu ${ }^{20}$ \\ (Affiliations can be found after the references)
}

Received 31 July 2013 / Accepted 22 November 2013

\begin{abstract}
Context. The Cluster Lensing And Supernovae survey with Hubble (CLASH) is a Hubble Space Telescope (HST) Multi-Cycle Treasury programme that observes 25 massive galaxy clusters, 20 of which were X-ray-selected to preferably choose dynamically relaxed clusters, and 5 additional "high magnification" clusters, which were selected based on their optical lensing properties. CLASH aims to study the dark matter distribution of the clusters and find magnified high-redshift galaxies behind them. CLASH observations were carried out in 16 bands from UV to NIR to derive accurate and reliable estimates of photometric redshifts.

Aims. We present the CLASH photometric redshifts using 16 HST bands and study the photometric redshift accuracy including a detailed comparison between photometric and spectroscopic redshifts for the strong lensing arcs using the measurements from the cluster MACSJ1206.2-0847. Methods. We used the publicly available Le Phare and BPZ photometric redshift estimation codes on 17 CLASH galaxy clusters for which the full photo- $z$ data processing had been completed at the time of this analysis, and derive an estimate of the CLASH photo- $z$ accuracy.

Results. Using Le Phare code for objects with a $S / N \geq 10$, we reach a precision of $3 \%(1+z)$ for the strong lensing arcs, which is reduced to $2.4 \%(1+z)$ after removing outliers. For galaxies in the cluster field, the corresponding values are $4 \%(1+z)$ and $3 \%(1+z)$. Using mock galaxy catalogues, we show that $3 \%(1+z)$ precision is what is expected using the baseline sky substraction algorithm when taking into account extinction from dust, emission lines, and the finite range of SEDs included in the photo- $z$ template library. An improved method for estimating galaxy colours that yields more accurate photometric redshifts will be explored in a forthcoming paper. We study photo- $z$ results for different aperture photometry techniques and find that the SExtractor isophotal photometry works best. We check the robustness of the arcs photo- $z$ results by rederiving the input photometry in the case of MACS1206. We describe and release a photometric redshift catalogue of the MACS1206 cluster we study.

Conclusions. Our photo- $z$ codes give similar results for the strong lensing arcs, as well as for galaxies of the cluster field. Results are improved when optimizing the photometric aperture shape that shows an optimal aperture size around 1" radius, giving results that are equivalent to isophotal photometry. Tailored photometry of the arcs improves the photo- $z$ results by showing more consistency between the different arcs of the same strong lensing system.
\end{abstract}

Key words. galaxies: clusters: general - galaxies: distances and redshifts

\section{Introduction}

CLASH (Postman et al. 2012) ${ }^{1}$ aims to provide very robust constraints on the dark matter distribution (including the central mass concentration parameter) in 25 massive galaxy clusters by using 16-band imaging from the Hubble Space Telescope (HST) and wide-field imaging from the Subaru Telescope. These data allow joint constraints to be made on the mass profile and matter distribution using both strong and weak lensing measurements (Zitrin et al. 2012, 2011; Umetsu et al. 2012; Coe et al. 2012). A key requirement in modelling cluster gravitational lenses is the ability to distinguish background galaxies (those sources at greater redshift than the cluster that can be strongly or weakly lensed) from the foreground galaxies (at lower redshifts than the cluster) and from the cluster member galaxies. The cluster

\footnotetext{
* Appendices are available in electronic form at http: //www . aanda.org

$\star \star$ Photometric redshift catalogue is only available at the CDS via anonymous ftp to cdsarc.u-strasbg. fr (130.79.128.5) or via http://cdsarc.u-strasbg.fr/viz-bin/qcat?]/A+A/562/A86

1 http://www. stsci . edu/ postman/CLASH/Home.html
}

member galaxies are important in establishing an initial guess at the cluster mass model. Good redshift estimates are required for both the cluster members and the background galaxies in order to measure the relative distance ratios between the lens and the sources - a key input into the lens mass modelling process. The 16-band HST imaging obtained in CLASH allows, in principle, superb photometric redshift estimates to be measured down to flux levels well beyond the reach of spectrographs on groundbased telescopes. In this paper, we quantify the accuracy of the CLASH photometric redshifts as well as discuss the accuracy required to reliably measure the cluster mass distributions. We will show that the two accuracies are in good agreement.

Redshift estimates are a necessary ingredient for gravitational lensing analyses, both weak and strong. Apart from identifying galaxies located behind the lensing cluster itself, the strength of the lensing signal depends on the ratio of the distances between lens and source galaxy and between the observer and the source, $D_{\mathrm{LS}} / D_{\mathrm{S}}$. In the strong lensing regime, accurate redshifts of each individual source are needed both to identify possible counter-images and to constrain the lensing mass model. For weak lensing, the mass model depends on an 
unbiased estimate of the mean distance ratio $\left\langle D_{\mathrm{LS}} / D_{\mathrm{S}}\right\rangle$ averaged over the background sources. Removing interlopers from the sample of background sources is paramount.

Most of the galaxies which are interesting for the lensing measurement both strong and weak are too faint for spectroscopic observations. Photometric redshifts have proved to be useful and essential tools for a range of astronomical applications, from wide-field galaxy surveys (e.g. the Dark Energy Survey $^{2}$, the Large Synoptic Survey Telescope ${ }^{3}$, and Euclid ${ }^{4}$ ) to targeted deep field observations such as the COSMOS survey (Scoville et al. 2007; Ilbert et al. 2009). There are several different publicly available photo- $z$ codes which, broadly speaking, can be categorized in two groups: empirical training and template matching methods. The empirical training methods include neural networks such as ANNz (Collister \& Lahav 2004; Vanzella et al. 2004), decision trees such as ArborZ (Gerdes et al. 2010), kd-trees (Csabai et al. 2007). Training methods require a statistically significant and representative redshiftmagnitude-colour training sample. Template fitting methods, such as Hyper-z (Bolzonella et al. 2000), BPZ (Benítez 2000), Le Phare (Ilbert et al. 2006), and EAZY (Brammer et al. 2008), require a representative library of galaxy spectral energy distributions (SEDs) that be fitted to the observations to find the best combination of redshift and template. The SED library can either be empirically (Coleman et al. 1980) or theoretically (Bruzual \& Charlot 2003) based.

There is no empirical training set available for the CLASH data and hence we make use of two template-based codes, BPZ and Le Phare. Hildebrandt et al. (2010) compared the photo- $z$ performances on simulated and real data and both BPZ and Le Phare compared favorably.

There have been earlier studies of galaxy photometric redshift in cluster environment such as Pelló et al. (2009); Guennou et al. (2010). Guennou et al. (2010) reaches a precision of $\sigma \approx 0.05\left(\mathrm{NMAD}^{5}\right)$ at $m(I)<24.5$ using 5 optical groundbased broadbands BVRIZ complemented with Spitzer IRAC 3.6 and $4.5 \mu \mathrm{m}$ and F814W ACS/HST observations. Their study shows a degradation in the photometric redshift accuracy for intrinsically bright galaxies and for the latest and earliest galaxy types in galaxy clusters. Guennou et al. (2010) showed an environmental dependence of the photo- $z$ accuracy which is interpreted as the photo- $z$ template being not well suited for galaxy clusters as quoted. Pelló et al. (2009) derived photo- $z$ for the ESO Distant Cluster Survey (EDisCS) fields (White et al. 2005) which is a sample of 20 clusters. They reach a precision $\sigma \approx 0.05$ to 0.06 at $I<22$ with respectively 4 and 5 optical and near-infrared (NIR) ground-based broadbands $B V I K$ s and VRIJKs for respectively a low- $z$ and high- $z$ cluster sample. The scope of these papers was to present their photo- $z$ quality with applications to studies of galaxy evolution for Pelló et al. (2009) and weak-lensing tomography for Guennou et al. (2010). In this paper, we study the accuracy of photo-z's based on HST photometry of both the cluster member galaxies and the lensed arcs identified as part of the CLASH strong lensing analysis.

The paper is structured as follows: in Sect. 2, we give a quick overview of the scientific goals and observations of the CLASH programme and specify the role and impact of the photo- $z$. Section 3 presents the two photo- $z$ techniques we use and we study their performance in Sect. 4. In Sect. 4.4, we compare the

\footnotetext{
2 http://www.darkenergysurvey.org/

3 http://www. lsst.org/lsst/

4 http://sci.esa.int/euclid/

$51.48 * \operatorname{median}\left[\Delta z_{\mathrm{p}} /\left(1+z_{\mathrm{s}}\right)\right]$.
}

Table 1. HST camera, band, magnitude limits for extended sources at $5 \sigma$ and completeness for the CLASH observations.

\begin{tabular}{lccc}
\hline \hline Camera & \# Filter & $A B \operatorname{mag}(5 \sigma)$ & $\% S / N>5$ \\
\hline WFC3/UVIS & $F 225 W$ & 24.8 & 69.3 \\
WFC3/UVIS & $F 275 W$ & 24.9 & 70.6 \\
WFC3/UVIS & $F 336 W$ & 25.2 & 79.0 \\
WFC3/UVIS & $F 390 W$ & 26.1 & 92.6 \\
ACS/WFC & $F 435 W$ & 26.2 & 94.3 \\
ACS/WFC & $F 475 W$ & 26.7 & 95.6 \\
ACS/WFC & $F 606 W$ & 27.2 & 98.6 \\
ACS/WFC & $F 625 W$ & 26.6 & 98.6 \\
ACS/WFC & $F 775 W$ & 26.5 & 99.7 \\
ACS/WFC & $F 814 W$ & 27.5 & 99.6 \\
ACS/WFC & $F 850 L P$ & 26.2 & 99.5 \\
WFC3/IR & $F 105 W$ & 27.0 & 96.2 \\
WFC3/IR & $F 110 W$ & 27.6 & 84.8 \\
WFC3/IR & $F 125 W$ & 27.1 & 86.7 \\
WFC3/IR & $F 140 W$ & 27.3 & 95.1 \\
WFC3/IR & $F 160 W$ & 27.2 & 98.0 \\
\hline
\end{tabular}

Notes. The magnitudes limits are an average over galaxies of different sizes. The filter transmission curves are shown in Fig. 1.

performance to an idealized scenario based on mock galaxy catalogues. Section 5 discusses issues with obtaining photo-z's for strongly lensed multiple images. We focus on MACS1206 as it has a high numbers of arcs and spectroscopic redshifts. Finally we present a photo- $z$ catalogue for MACS1206 which includes both BPZ and Le Phare photo-z.

Throughout this paper we assume a flat Lambda-CDM cosmology with $\Omega_{\mathrm{m}}, \Omega_{\Lambda}=(0.3,0.7)$ and use the $A B$ magnitude system.

\section{The CLASH programme}

CLASH is an HST Multi-Cycle Treasury programme. Observations in the 16 HST broad passbands have been completed for the 25 clusters.

\subsection{The CLASH photometry}

The photometry is the most critical issue for photo- $z$ accuracy. This section gives an overview of the photometric quality and completeness of the CLASH data and catalogues. For more details, the reader should refer to (Postman et al. 2012) Sect. 5.

Image co-addition and alignment onto a grid with $0.065^{\prime \prime}$ per pixel is performed with an automated pipeline based on MosaicDrizzle (Koekemoer et al. 2011). Object detection and photometry is then accomplished using SExtractor in dual image mode using a weighted sum of the ACS/WFC and WFC3/IR images (Bertin \& Arnouts 1996).

The photometry is measured in isophotal apertures and corrected for galactic extinction using Schlegel et al. (1998). A local sky background is estimated for each object within an annulus of 26 pixels. We do not model and subtract the galaxy cluster light from the standard pipeline products. The impact of a more advanced cluster light sky subtraction algorithm on the CLASH photometric redshift accuracy will be investigated in separate paper (Molino et al., in prep.).

In Table 1 we list the filters used for the CLASH observations with their $5 \sigma$ depths derived from all the CLASH clusters sample and the percentage of galaxies observed for each filter 


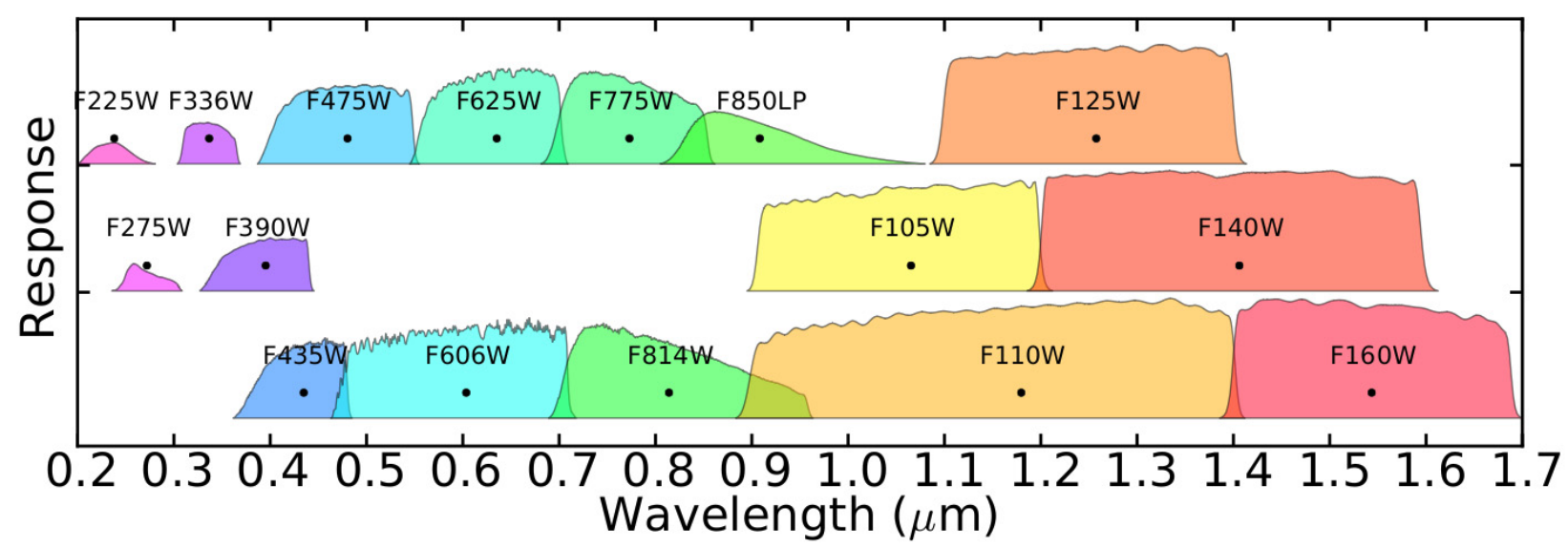

Fig. 1. HST filter set used by CLASH, showing the transmission as a function of wavelength from UV to NIR.

down to $S / N(F 814 W)=5$ in MACS1206. The magnitude limits shown are not the point source limits but rather the mean limits averaged over all extended sources (i.e., galaxies) without regards to size. Magnitude limits based on point sources can be found in Postman et al. (2012).

\subsection{CLASH spectroscopic redshifts}

We use a sample of spectroscopic redshifts (spec-z's) which were primarily acquired as part of our VIMOS/VLT Large Program 186.A-0798, targeting the 14 southern hemisphere CLASH clusters (Rosati, in prep.) (for the dynamical mass profiles of MACS1206, see Lemze et al. 2013; and Biviano et al. 2013). This programme targets many thousands of galaxies around CLASH clusters drawn from our wide-field Subaru SuprimeCAM imaging data. In this paper, we use the 689 galaxies that have a spectroscopic redshift at least $80 \%$ secure and that also lie within the narrower CLASH/HST field of view that contains our 16-band imaging data. In addition, we have included spec-z's from the literature and from observations at the Magellan Telescope and the MMT.

The spec-z's span a magnitude range of $18<m(F 814 W)<26$ as shown in Fig. 2. The spec- $z$ sample has a higher fraction of red sequence galaxies than a randomly chosen galaxy sample since one of the VLT programme's primary objectives is cluster dynamics. Lensed arcs have also been targeted but they do not represent a large fraction of the spec- $z$ sample. We use the spec- $z$. sample only to assess the photo- $z$ accuracy but do not use it for spectral template calibration.

Following Maturi et al. (2013), we divide the spec- $z$ sample into two: a low-redshift sample containing 584 galaxy cluster members and foreground galaxies $\left(z_{\mathrm{s}} \leq z_{\text {cluster }}+0.2\right)$, and a highredshift sample containing 106 lensed background galaxies $\left(z_{\mathrm{s}}>\right.$ $z_{\text {cluster }}+0.2$ ). In both samples, we only use redshifts considered to be at least $80 \%$ secure.

\section{Photometric redshift estimation}

As mentioned above, we make use of two template-based photo- $z$ codes: Le Phare and BPZ. In this section, we briefly describe these codes as well as their validity for application to the CLASH/HST data.

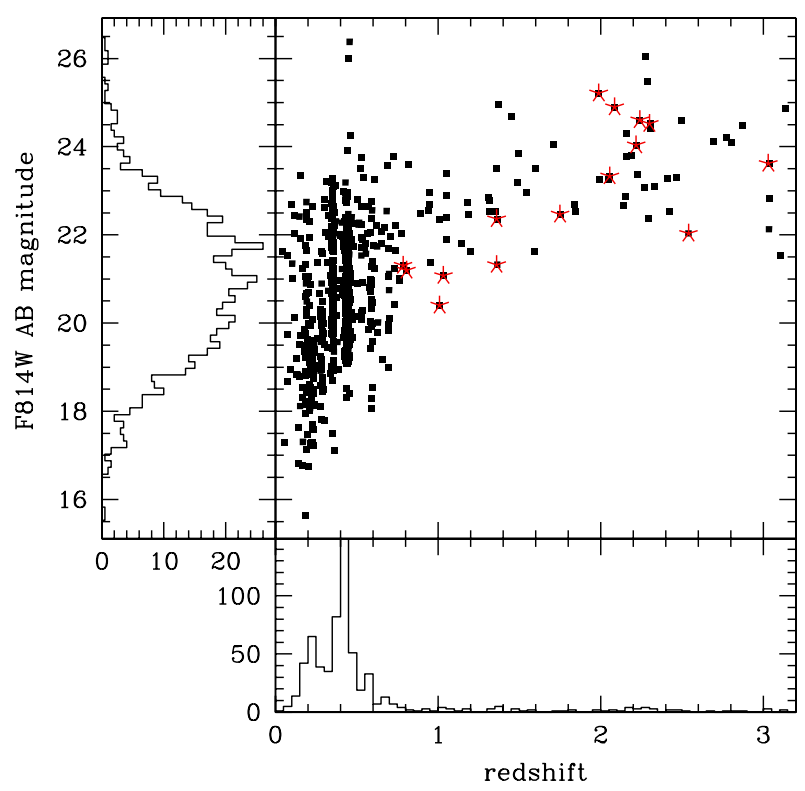

Fig. 2. Spec- $z$ sample we used to assess the photo- $z$ quality. Magnitude $(F 814 W)$ and spectroscopic redshift from 17 cluster fields. Most spec- $z$ are either lensed galaxies at $z_{\mathrm{s}}>1$, or galaxy cluster members at $z_{\mathrm{s}}<1$. The red stars show the obvious strong-lensing arcs for which we have a spectroscopic redshift.

\subsection{Le Phare}

Le Phare ${ }^{6}$ is a public photo- $z$ code based on a template fitting method used in Ilbert et al. $(2006,2009)$. It uses a $\chi^{2}$ minimization defined as:

$\chi_{\text {model }}^{2}=\sum_{i=1}^{n}\left(\left[F_{\text {obs }}^{i}-\alpha F_{\text {model }}^{i}\right] / \sigma^{i}\right)^{2}$,

where $F_{\text {obs }}^{i}$ and $F_{\text {model }}^{i}$ are the measured and the template model fluxes in filter $i$ and $\sigma^{i}$ is the corresponding measured flux error. We use the templates optimized for the COSMOS photo- $z$ library as described in Ilbert et al. (2009). This library is composed of 31 templates ranging from ellipticals to starburst types. There are three elliptical galaxy templates and seven spiral galaxy templates generated by Polletta et al. (2007). In addition, there

6 http://www. cfht .hawaii.edu/ arnouts/lephare.html 
are 12 templates of starburst galaxies from Bruzual \& Charlot (2003) with ages between 0.03 and 3 Gyr. For the intermediate and late-type populations, we apply extinction law from Calzetti et al. (2000) with extinctions values of $E(B-V)=0.1,0.2,0.3$, $0.4,0.5$. Le Phare does not interpolate between templates. We add emission line fluxes to the magnitudes derived in the template library. This is a standard procedure from Le Phare aiming to better reproduce the colours of galaxies with significant ongoing star formation, as described in Ilbert et al. (2009); Jouvel et al. (2009).

\section{2. $B P Z$}

BPZ $^{7}$ (Benítez 2000; Benítez et al. 2004; Coe et al. 2006), is a Bayesian photo- $z$ estimation based on a template fitting method. BPZ uses a new library (Benitez, in prep.) composed by five SED templates originally drawn from PEGASE (Fioc \& Rocca-Volmerange 1997) that were adjusted and recalibrated from FIREWORKS photometry and spectroscopic redshifts (Wuyts et al. 2008) to optimize its performance. The FIREWORKS data includes photometry for galaxies down to 24.3 ( $5 \sigma K$ band) out to $z \sim 3.7$ from 0.38 to $24 \mu \mathrm{m}$. In analyses of large data sets with high quality spectra, this template set yields less than $1 \%$ outliers, demonstrating that it emcompasses the range of metallicities, extinctions, star formation histories observed for the vast majority of galaxies. These templates include nebular emission lines as implemented by Fioc \& Rocca-Volmerange (1997). In addition, the BPZ library also includes five templates from GRASIL and a starburst galaxy. BPZ library is then composed by 11 galaxy templates and different sets of priors based on redshift, types, and magnitude distribution from existing surveys and allows for interpolation between templates in the $\chi^{2}$ minimization.

$\mathrm{BPZ}$ is the default method used in the pipeline to derive photo- $z$ for CLASH.

\subsection{Definition of photo-z quality}

To assess the quality of the CLASH photo- $z$, we compute the scatter, mean, and outliers number of the photo- $z$ deviation, $\Delta z_{\mathrm{p}}=z_{\mathrm{p}}-z_{\mathrm{s}}$. The scatter is measured by the normalized median absolute deviation (NMAD) described in Hoaglin et al. (1983), which is given by $1.48 * \operatorname{median}\left[\Delta z_{\mathrm{p}} /\left(1+z_{\mathrm{s}}\right)\right]$. This is a robust estimator, less liable to biasing by catastrophic outliers than the sample standard deviation. The mean $(\mu)$ is the mean value of $\Delta z_{\mathrm{p}} /\left(1+z_{\mathrm{s}}\right)$ and the number of outliers $(\eta)$ is defined as galaxies whose $\left|\Delta z_{\mathrm{p}}\right| /\left(1+z_{\mathrm{s}}\right)>0.15$.

We select sub-samples of the photo- $z$ based on estimates of the reliability of each photo- $z$ (called $o d d s$ for BPZ and $p d z \_$best for Le Phare). This parameter is the integral around the best-fit redshift of the probability distribution of the redshift $p(z)$ given by the $\chi^{2}$ fitting. We take a window of 2.5 times the photo- $z$ precision expected from mock catalogue for the CLASH survey which is a window of 0.05 around the best-fit redshift. The odds is a number between 0 and 1 which is equal to 1 if the $p(z)$ has a single, well-defined narrow peak, while it will be at lower value if there are multiple peaks or if the peak is broader than 0.05 in redshift.

\footnotetext{
7 http://www.its.caltech.edu/ coe/BPZ/
}

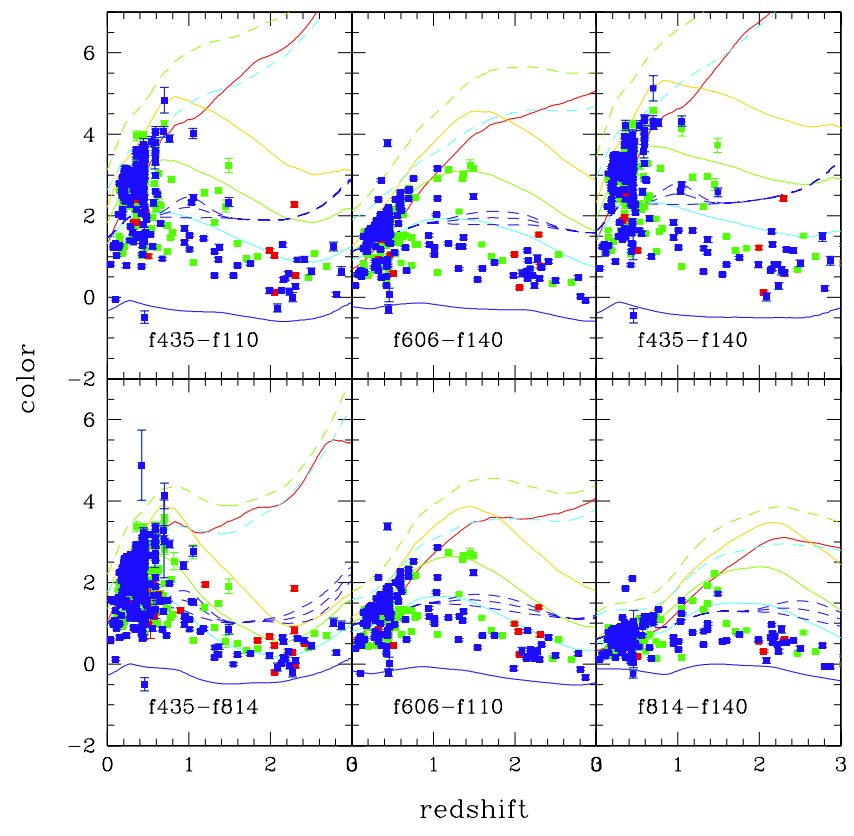

Fig. 3. HST colour as a function of galaxy redshift. The solid and dashed lines show the COSMOS library colours intrinsic and extended using Calzetti extinction law. While the library is composed with 31 models, we show two ellipticals (red-gold), one intermediate (green) and two starbursts (cyan-blue). The blue, green and red squares correspond to the observed colours of the spectroscopic redshift sample. The blue squares are the galaxies with good photometric redshift $2 \%(1+z)$, the red squares are catastrophic outliers $(>15 \%(1+z))$ for which odds are higher than $90 \%$. The green squares are the rest of the sample. The photo- $z$ library colours match the colour of CLASH observations well.

\subsection{Template library representation}

To derive good photo-z's one requires a template library that contains spectra that are representative of the galaxy types and colours observed by CLASH. The BPZ and Le Phare redshift estimates are based on $\chi^{2}$ minimizations respectively with and without the help of sets of priors and template combination.

The CLASH spec- $z$ sample of 689 galaxies contains mainly galaxies with $z_{\mathrm{s}}<1$. In this range, the strongest colour gradient, from the Balmer/D4000 breaks, will happen in between $F 435 \mathrm{~W}$ and $F 814 W$. Figure 3 shows different colours as a function of redshift. The COSMOS library is represented by the solid and dashed lines and the CLASH/HST spec-z sample is represented by the dots. It is clear that the library provides good coverage of the spec- $z$ sample.

\section{CLASH photo-z accuracy}

In this section, we study the photo- $z$ accuracy and number of outliers for the galaxies in the foreground and cluster members, for the lensed background galaxies, and the full spec-z sample as explained in Sect. 2.2. First, we study the overall accuracy for all 16 filters with Le Phare and BPZ then compare different subsets of filters. Next, we investigate photo- $z$ accuracy based on fixed-aperture and the automated Kron photometry (mag_auto) and, finally, we discuss the photo- $z$ performance in an idealized scenario based on mock catalogues. 


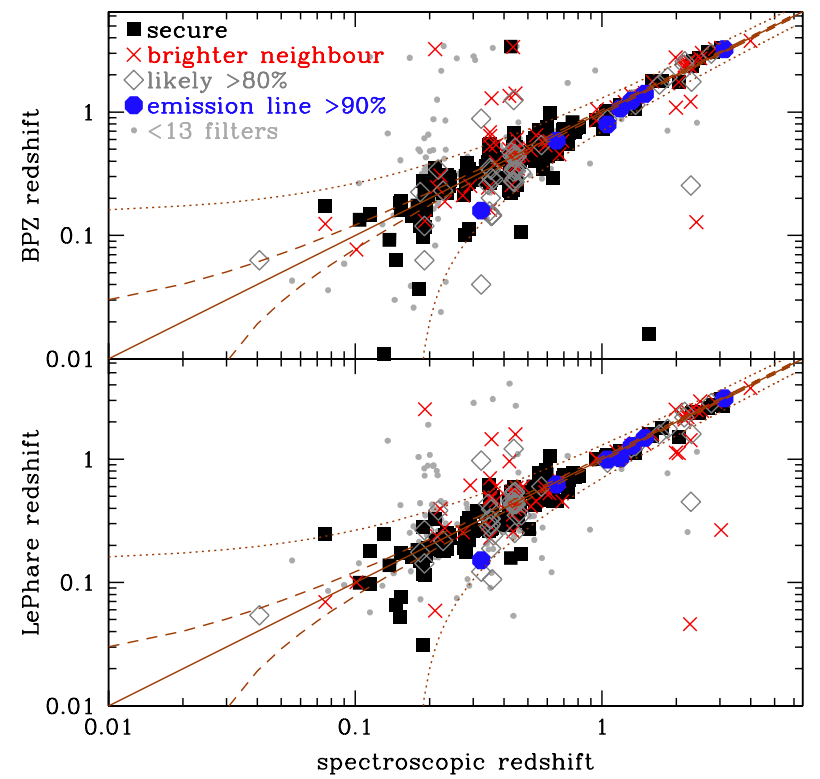

Fig. 4. Spectroscopic vs. photometric redshifts for the CLASH spec- $z$ sample showing Le Phare (bottom) and BPZ (top) performances for the spec- $z$ secure redshift in black squares, likely redshift in dark grey diamonds and one emission line redshift in blue hexagons observed in at least 13 filters. We also added galaxies with a number of detections in less than 13 filters in light grey dots. The red crosses mark the spec- $z$ for which the closest HST photometry match had a possible other solution at less than $3^{\prime \prime}$. The brown dashed, and dotted curves correspond to $z_{\mathrm{p}}=z_{\mathrm{s}} \pm 0.02\left(1+z_{\mathrm{s}}\right)$, and $z_{\mathrm{p}}=z_{\mathrm{s}} \pm 0.15\left(1+z_{\mathrm{s}}\right)$.

\subsection{Photometric redshift comparison: BPZ and Le Phare}

Now we compare the BPZ and Le Phare photo- $z$ estimates of the spec- $z$ sample. Figure 4 shows the photo- $z$ vs. spec- $z$ for the full spec- $z$ sample. For Le Phare photo- $z$ estimation, we include the zeropoints or systematic shifts in the magnitudes. Table A.2 shows the shift's values that have been derived using the spec- $z$ sample up to magnitude $m(F 775 W)<24$. These shifts account for the mismatches between the CLASH colours and the photo- $z$ library colours and possible problems, if any, on our transmission (filter-mirror-detector) throughput model. The shifts are less than a tenth of a magnitude for the optical and NIR filters and can go up to $0.4 \mathrm{mag}$ for the UVIS filters. The variance of the zeropoints in the UVIS shifts is large, especially for the filters $F 225 \mathrm{~W}$ and $F 275 \mathrm{~W}$. The spec- $z$ sample is dominated by galaxies at $z<0.8(90 \%)$ and there is either a weak detection or no detection in the UVIS filters. In the case of the UVIS filters, incompleteness in the template libraries is expected to be the main contributor to the variance in the zeropoint estimation, in particular the reduced number of star-forming galaxy templates. In addition, interpolation between the templates is not allowed in Le Phare. Dust extinction also adds degeneracies to the zeropoint computation. These last factors make the zeropoint computation in the NUV wavelenghts more challenging.

\subsubsection{Photometric redshift results}

Figures 4 and 5 show BPZ and Le Phare photo- $z$ vs. spec- $z$ for galaxies observed in at least 13 filters. Both methods show very good results for galaxies with secure spec-z's. There are only a few significant outliers (as defined in Sect. 3.3). Some of these have brighter galaxies (or galaxy segments) nearby (within $3^{\prime \prime}$ ). Light from these brighter neighbors may have
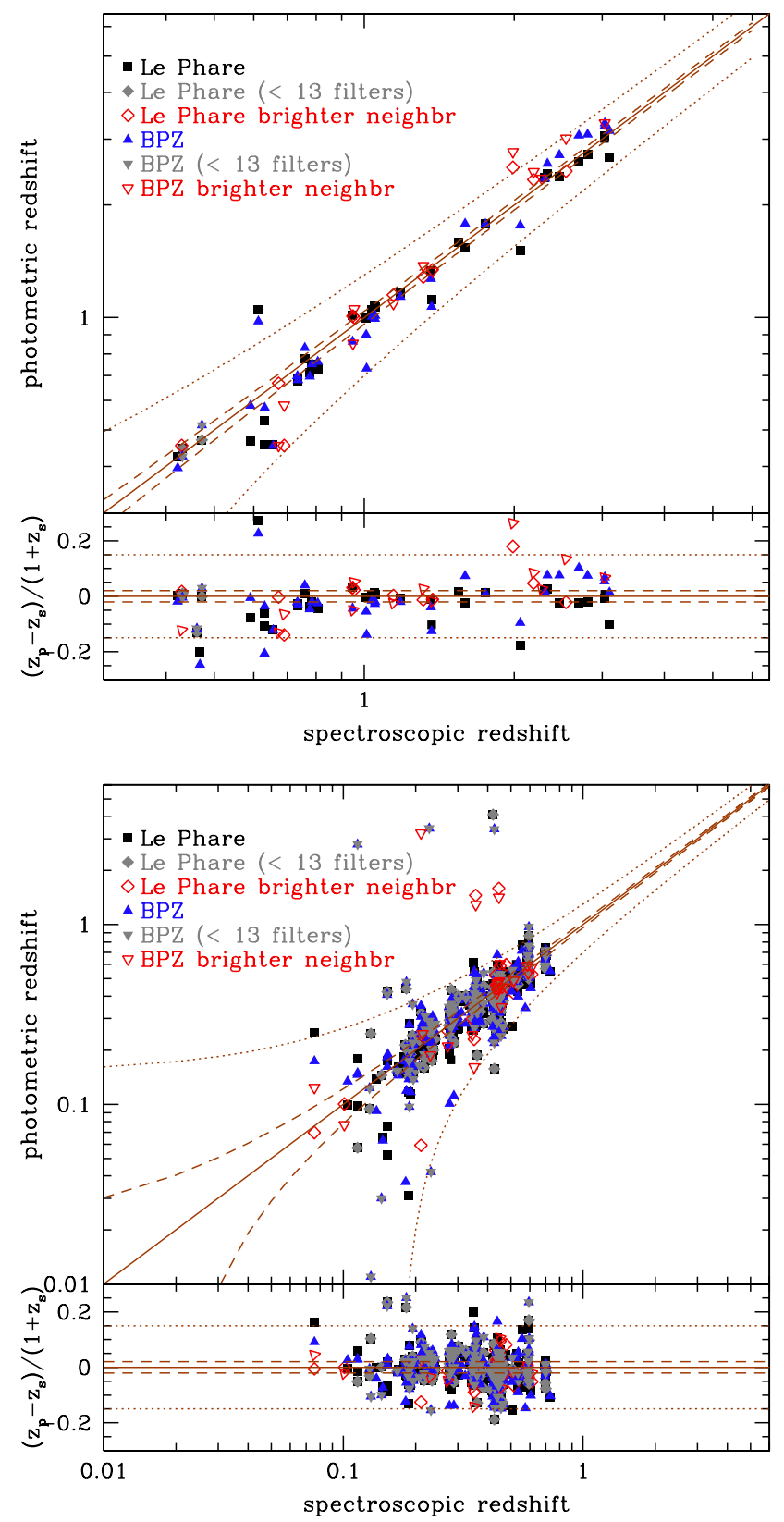

Fig. 5. Spectroscopic vs. photometric redshifts for the CLASH spec- $z$ sample whose magnitude and redshift distributions are shown in Fig. 2. The black squares and blue triangles are the Le Phare and BPZ photo- $z$ for galaxies observed in at least 7 bands with secure spec- $z$. The grey triangles and grey diamonds are respectively BPZ and Le Phare secure redshifts observed in less than 13 filters. The empty red triangles and diamonds are the spec- $z$ with a brighter neighbour within $3^{\prime \prime}$ of the closest match to the spec-z. The top figure corresponds to galaxy lensed images behind the cluster at a redshift of $z_{\mathrm{s}}>z_{\text {cluster }}+0.2$. The bottom figure corresponds to galaxy cluster members and foreground galaxies at $z_{\mathrm{s}} \leq z_{\text {cluster }}+0.2$.

entered the spectroscopic slits in some cases. Relative astrometric uncertainties of $1^{\prime \prime}$ between the spectroscopic and HST catalogues may also contribute to occasional incorrect crossmatching. Of the remaining significant outliers, one is at the edge of the WFC3/IR field of view and another has a spec- $z$ lacking a quality flag. In futher analysis, we will only use the 554 secure spec- $z$ (out of 689 total) to accurately assess the photo- $z$ quality.

We choose to look at galaxies observed in at least 7 filters to cover the optical wavelength range and with a 
signal-to-noise ratio $S / N>10$ in $F 775 W$. With the latter selections we have a sample of 63 lensed background galaxies and 327 foreground-cluster galaxies. On these galaxy samples, BPZ and Le Phare have good performances for foreground and cluster member galaxies with an NMAD of 3 to $4 \%(1+z)$, respectively, with and without photo- $z$ quality cuts. For the lensed sample, we achieve a precision of 2.4 to $3 \%$ for Le Phare when using the photo- $z$ estimations which include systematic shifts. Without systematic shifts, BPZ and Le Phare have similar photo- $z$ scatter with an NMAD of $5 \%(1+z)$. Figure 5 show Le Phare photo- $z$ with systematic shifts and BPZ for lensed background galaxies and foreground-cluster galaxies. Over the whole redshift range, both Le Phare and BPZ have separately 18 catastrophic outliers. One could remove 7 out of the 18 outliers in discarding galaxies which have different values for Le Phare and BPZ photo- $z$. For the 11 other outliers, both BPZ and Le Phare have very close photo- $z$ estimations. As explained in the latter section, catastrophic outliers are either galaxies observed in less than 13 filters, unsecure spec- $z$ or a mismatch of the spec- $z$. We note that the source background galaxies have less outliers than the foreground and cluster galaxies. Most galaxies living inside clusters have red colours from their old star population. Photo- $z$ libraries do not include the reddest galaxies such as the BCG. To improve further our photo- $z$ estimations, there are ongoing efforts to create better templates for galaxies in cluster environments (e.g. Greisel et al. 2013). Including these models would improve the photo- $z$ for cluster members. In Sect. 5, we study the photometry and photo- $z$ of the lensed images for one CLASH cluster, using customized photometry to have more control on the aperture shape in order to achieve a better $\mathrm{S} / \mathrm{N}$ and have more accurate colours for the photo- $z$ estimations.

\subsubsection{Photometric redshift quality estimators}

In this section, we study the parameters that give an estimation of the photo- $z$ quality such as the $\chi^{2}$ and odds/pdz_best defined in Sect. 3, the 1 and $2 \sigma$ confidence region of the best-fit redshift. Figure 6 show the photo- $z$ precision as a function of the number of galaxies sorted using $\chi^{2}$ or odds $/ p d z$ best. According to Fig. 6, BPZ $\chi^{2}$ and Le Phare $p d z$ best give a good estimation of the photo- $z$ quality for their respective codes.

In order to study the reliability of the photo- $z$ estimation, we look at the $1-$ and 2- $\sigma$ confidence region. We compute the confidence region using the cumulative values of $\left|z_{\mathrm{p}}-z_{\mathrm{s}}\right|$. We find $62 \%$ of galaxies with $z_{\mathrm{p}}$ within $1 \sigma$ of $z_{\mathrm{s}}$ for Le Phare. We find respectively $96 \%$ and $90 \%$ of $z_{\mathrm{p}}$ within $2 \sigma$ of $z_{\mathrm{s}}$ for Le Phare and BPZ. To assess the reliability of these estimations, we bootstrap the 670 galaxies of our sample and find standard deviations of $0.61 \pm 0.02$ and $0.956 \pm 0.008$ for respectively the 1 and $2 \sigma$ confidence region of Le Phare. We find $0.906 \pm 0.011$ for BPZ $2 \sigma$ confidence region. However, these calculations assume that $p(z)$ is symmetric. We looked at $z_{\mathrm{p}}-z_{\mathrm{s}}$ and find $40 \%$ and $82 \%$ of $z_{\mathrm{s}}$ within the 1 and $2 \sigma$ Le Phare confidence region and $58 \%$ of $z_{\mathrm{s}}$ within BPZ $2 \sigma$ confidence region. The 1 and $2 \sigma$ regions are under-estimated for our spec- $z$ sample. Our spec- $z$ sample is mainly composed of bright galaxies which belong the galaxy clusters. The $p(z)$ of bright galaxies are usually narrow and do not follow a Gaussian profile. This result is then not unexpected. We note this could also come from doublepeaked $p(z)$ from the Lyman-Balmer break confusion. When using the photo- $z$ estimates, one should be careful in using a single best value photo- $z$ (mean or median of the $p(z)$ ) and rather use

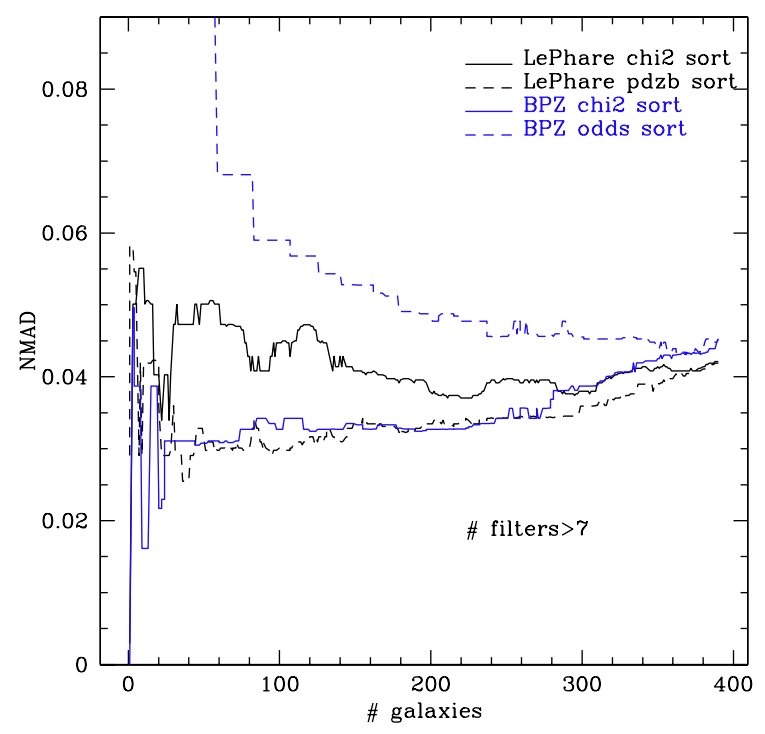

Fig. 6. Photo- $z$ precision for the CLASH spec- $z$ sample as a function of the number of galaxies sorted using BPZ odds/Le Phare pdz_best (dashed lines) or $\chi^{2}$ (solid lines) from the photoz fit. Blue and black lines show respectively BPZ and Le Phare results on the sample of galaxies observed in at least 7 filters.

Table 2. Characteristics of the photo- $z$ distribution for sub-sets of the CLASH filters described in the above paragraphs.

\begin{tabular}{lccc}
\hline \hline Photometry & NMAD & $\mu\left[\Delta z_{\mathrm{p}} /\left(1+z_{\mathrm{s}}\right)\right]$ & $\eta$ \\
\hline "ACS" & 0.051 & -0.006 & 19 \\
"UVIS_ACS" & 0.051 & -0.020 & 18 \\
"ACS_NIR" & 0.044 & -0.009 & 16 \\
"UVIS1_ACS_NIR" & 0.041 & -0.013 & 9 \\
"UVIS2_ACS_NIR" & 0.039 & -0.010 & 8 \\
"UVIS_ACS_NIR" & 0.040 & -0.012 & 8 \\
\hline
\end{tabular}

Notes. We use galaxies observed in at least 13 filters with a $S / N>10$ in $F 775 \mathrm{~W}$ resulting in 303 galaxies or $44 \%$ of the spec- $z$ sample. The first column is the sub-set of filters in which we compute photo- $z$, the second and third columns are the NMAD and the median of the $z_{\mathrm{p}}-z_{\mathrm{s}}$ distribution. The last column $\eta$ is the number of catastrophic outliers.

the full $p(z)$ distribution. The $p(z)$ captures the whole information output by photo- $z$ codes which help to decrease biaises.

\subsection{Photometric redshift and systematics from the photometry}

We select galaxies observed in at least 13 filters with a $S / N>10$ in $F 775 \mathrm{~W}$ without selection in magnitude or redshift. We do not select on the quality of the photo- $z$ fits (odds), in order to have identical galaxy samples for fair comparisons between sub-samples. This resulting sub-sample contains 289 galaxies that represents $42 \%$ of the spec- $z$ galaxy sample with full HST coverage.

We show the utility of all filters especially for the range of redshift for which we want accurate photo- $z$. We compute photo- $z$ for the six following sub-samples of filters:

- optical filters of the ACS camera;

- NUV+optical filters of the WFC3/UVIS+ACS cameras;

- ACS+NIR filters of the ACS+WFC3/IR camera;

- UVIS1 $(F 390 W)+\mathrm{ACS}+\mathrm{NIR}$ of ACS+WFC3; 


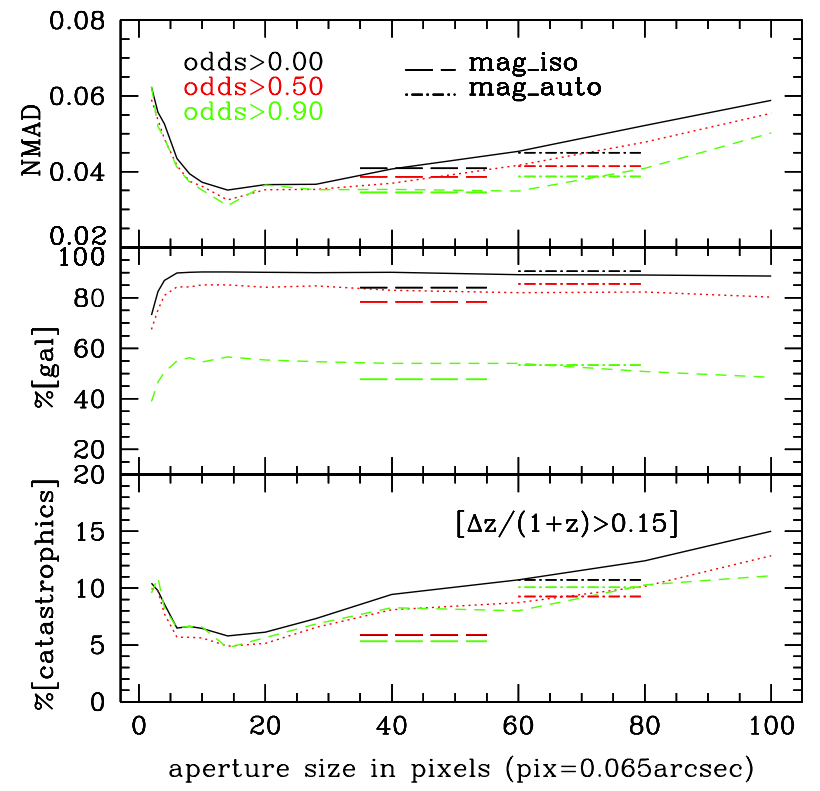

Fig. 7. Top panel: photo- $z$ scatter, middle panel: percentage of galaxies selected and bottom panel: percentage of catastrophic outliers for various choices of photometric apertures, using the full spec- $z$ sample. The solid black, dotted red, long-dashed green lines show the results at $p d z$ best cut of $0,0.5,0.9$. The optimal fixed aperture size is around 10-30 pixels. Isophotal photometry (long-dashed lines) performs close to equally well this range of fixed apertures. Automated Kron apertures (dash-dotted lines) are not competitive.

\section{- UVIS2 $(F 336 W+F 390 W)+\mathrm{ACS}+\mathrm{NIR}$ of ACS+WFC3; \\ - NUV+optical+NIR filters of ACS+WFC3.}

Table 2 shows the scatter, median and number of catastrophic outliers for the different sub-samples of the CLASH filters mentioned above as defined in Sect. 3.3. The full filter set yields a scatter of $4.1 \%(1+z)$, and 8 "catastrophic" outliers as defined in Sect. 3.3 (where $\left.\left|z_{\text {phot }}-z_{\text {spec }}\right| /\left(1+z_{\text {spec }}\right)>0.15\right)$. Removing the two bluest filters of the UVIS camera (UVIS2+ACS+NIR) reduces the scatter to $3.9 \%(1+z)$. We do not expect the 2 UVIS filters to significantly improve photo- $z$ accuracy for galaxies at redshift lower than 0.8 , only at redshifts greater than 0.8 . The bluest CLASH filters help the photo- $z$ determination for galaxies at $z>0.8$ when the Lyman break becomes visible in the first UVIS filter $F 225 \mathrm{~W}$.

The photo- $z$ of Table 2 include systematic shifts in the photometry computed using the spec- $z$ sample. The shift's values are listed in Table A.2.

\subsection{Photometric redshift and aperture photometry}

Now we study the impact of different choices of photometry, including fixed apertures and automated Kron photometry.

We consider fixed apertures with diameters ranging from 2 pixels to 100 pixels (the pixel size is 0.065 arcsec in CLASH co-added images). We also measure Kron photometry (Kron 1980; Bertin \& Arnouts 1996), based on ellipsoidal apertures whose shape is calculated from the second moments of the light distribution. The Kron scaling factor was set to 2.5, and the minimum radius to be $3.3 \sigma_{\text {iso }}$ (the isophotal radius) which is in the range of values suggested by the SExtractor manual. We compare with the photo- $z$ based on the standard isophotal photometry using the entire spectroscopic redshift sample.

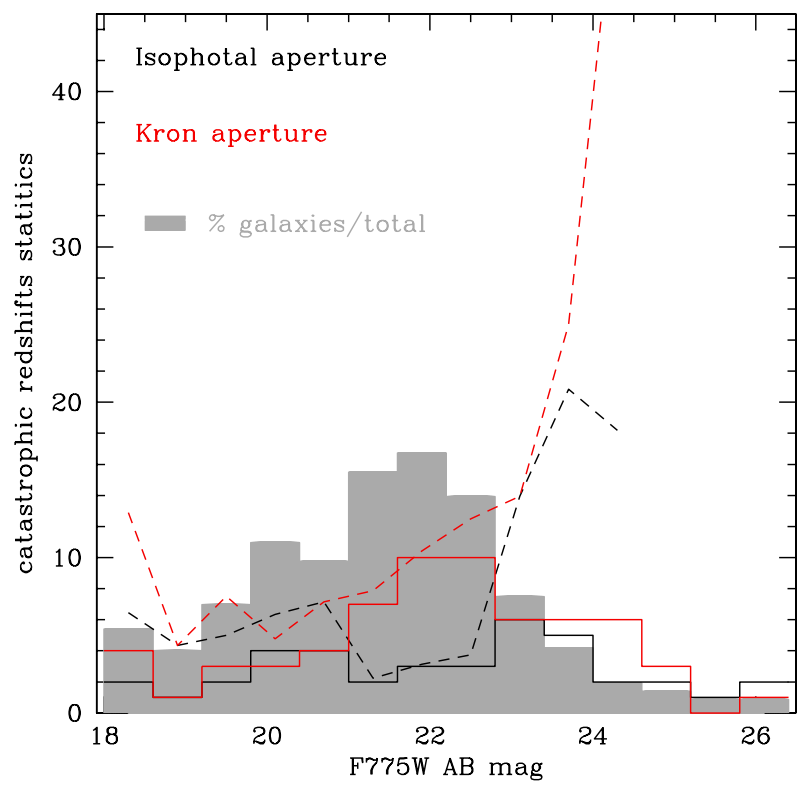

Fig. 8. Number and percentage of catastrophic outliers as a function of $F 775 \mathrm{~W}$ magnitude in solid and dashed lines respectively. We find 39 and 64 catastrophic outliers for respectively the isophotal and Kron aperture photometries. The grey shaded histogram shows the percentage of galaxies compared to the entire spec- $z$ sample in each magnitude bin.

Figure 7 top panel shows the photo- $z$ scatter for the different apertures. There is a range of aperture diameters where the colour is maximized and the error minimized and that range corresponds to roughly 10 to 30 pixels. Automated Kron apertures do not give very good results compared to a carefully chosen aperture photometry with a NMAD of $4.5 \%(1+z)$ for the highest $p d z \_$best cut. Standard isophotal photometry (threshold scale at $1 \sigma)$ gives the best results with a NMAD $3.0 \%(1+z)$. Figure 7 middle panel shows the percentage of galaxies that enters in the NMAD calculation of Fig. 7 top panel. Comparing the top and middle panel of Fig. 7, it is clear that the pdz_best cuts do not improve the scatter and number of outliers significantly when using the best range of aperture photometry. The bottom panel of Fig. 7 shows the percentage of catastrophic outliers as a function of aperture diameter. Again, fixed apertures of 10-30 pixels perform the best, minimizing the number of catastrophic outliers, optimizing the number of galaxies used, and pdz_best cuts do not improve the results significantly. Isophotal photometry gives very close results to the best range of fixed apertures.

Figure 8 shows the number and percentages of catastrophic outliers. We compared both the isophotal and automated Kron photometry. The isophotal photometry performs better than the Kron photometry with 39 catastrophic outliers compared to 64 for the Kron aperture using a catastrophic outliers definition defined in Sect. 3.3. It represents a percentage of $7 \%$ for the isophotal photometry and $11 \%$ for the Kron photometry. The percentage of catastrophic outliers by magnitude bins rises at magnitude $m(F 775 W)>23$. At $m(F 775 W)>23$ the percentage of galaxies compared to the entire sample lowers as shown by the grey shaded histograms. The percentages are thus not very significant. Figure 9 shows the $F 814 W$ magnitude error as a function of the colour $F 435 W-F 814 W$. Bottom left, top left, bottom right panels show the colour-photometric error relation for aperture diameters of 2, 14, and 100 pixels while the top right panel shows the corresponding isophotal photometry. The isophotal and 14 pixels aperture photometry optimize the colour 


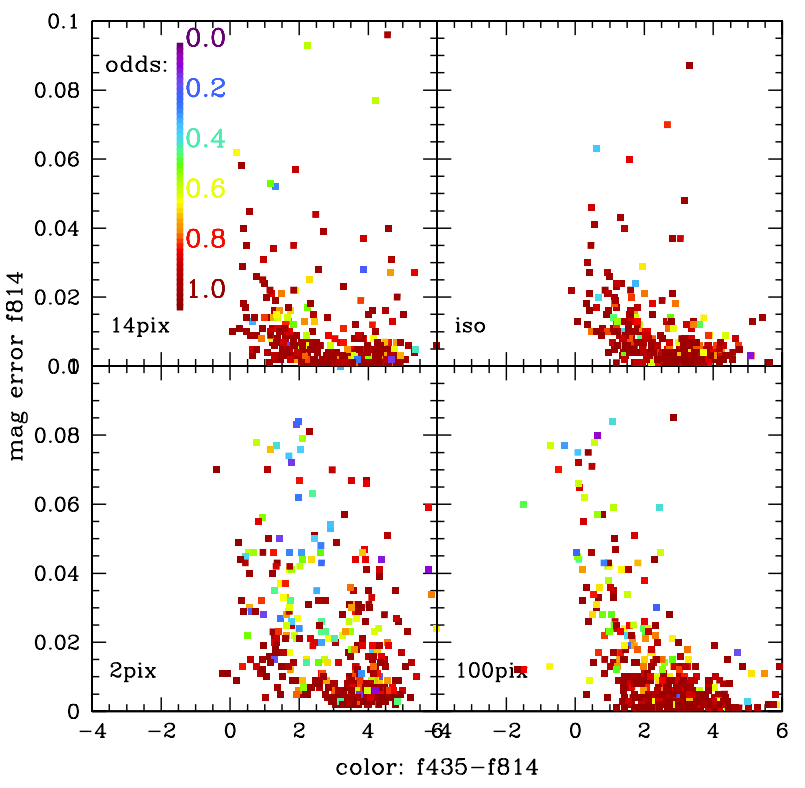

Fig. 9. Magnitude error as a function of galaxy colour is shown for aperture photometry done using 2, 14, and 100 pixel apertures in the lower left, upper left, and lower right panels, respectively. The mag errors as a function of colour for isophotal photometry is shown in the upper right panel. The 14 pixels aperture and isophotal photometry have higher colour accuracy than the 2 and 100 pixels aperture photometry, which explains the photo- $z$ results.

from the Balmer/D4000 break while minimizing the photometric error. In the case of a 2 pixels and 100 pixels photometry, the colour of the break is lost due to the lack of flux from small aperture size or the high level noise due to the big aperture size. We choose to use isophotal photometry for our studies.

We want to stress that these results have been computed using cluster galaxies. These results might change for smaller starburst galaxies at higher redshifts.

\subsection{Photometric redshifts from simulated CLASH photometry}

This section aims at testing the impact of systematics coming from galaxy properties on the photo- $z$ estimations from the CLASH photometry in an ideal case. We use catalogues from Le Phare simulations that are described in Jouvel et al. (2009).

We produce mock galaxy catalogues in the CLASH/HST bands based on a luminosity function that depends on both redshift and morphological type as derived from the GOODS survey (Giavalisco et al. 2004; Dahlen et al. 2005). The simulations do not include the background cluster light, characteristic of a cluster field, so this should be regarded as "best-case" scenario. The simulations are based on a mixture of templates which includes elliptical galaxies up to starbursts types. We miss the reddest galaxies such as the BCG's and the oldest galaxy population. This would likely affect the photo- $z$ accuracy of galaxies at $z_{\mathrm{s}}<1$. It should however impact less the photo- $z$ accuracy of the lensed background sample which have a higher fraction of blue young galaxies.

Input templates come from Coleman et al. (1980), extended in wavelength with synthetic spectra from Bruzual \& Charlot (2003). There are five main templates and one starburst template which are linearily interpolated to provide 66 independent characterizations of a galaxy's SED, which we refer to as the Coleman Extended (CE) templates. The luminosity function has
Table 3. Description of the simulations for which results are shown in Fig. 10.

\begin{tabular}{lll}
\hline \hline$X$ & Mock & Photo- $z$ \\
\hline Simul1 & CE & CE \\
Simul2 & CE+ext & CE+ext \\
Simul3 & CE+ext+eml & CE+ext+eml \\
Simul4 & CE+ext+eml & COSMOS syss \\
\hline
\end{tabular}

Notes. Each row represents a different simulation and photo- $z$ calculation, where the 2nd and 3rd columns are the library used in the mock catalogue construction and the photo- $z$ library, respectively. The +ext and $+\mathrm{eml}$ stands for the addition of extinction and emission lines to the libraries.

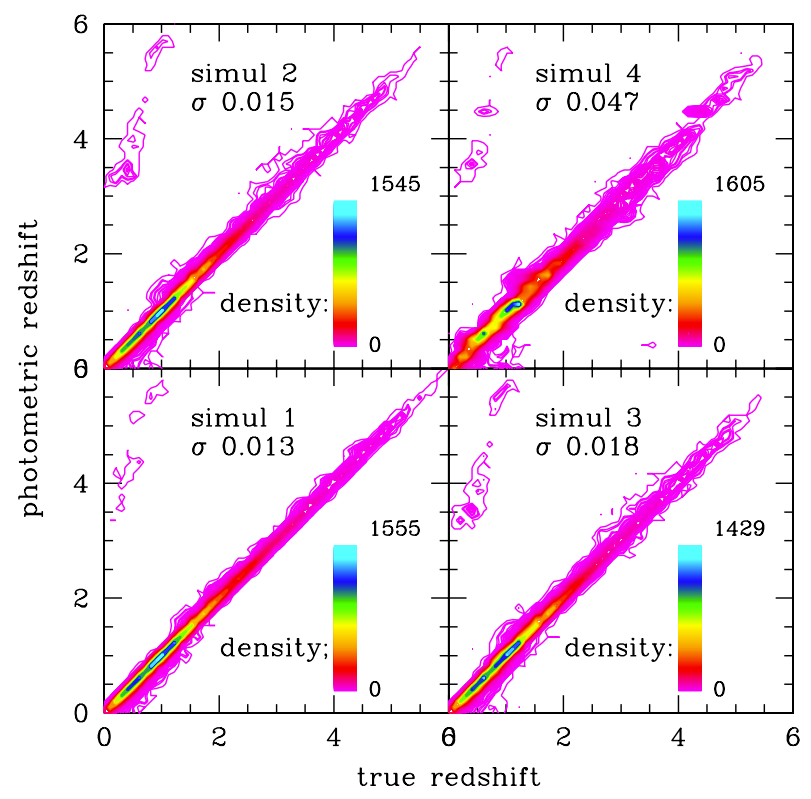

Fig. 10. Photometric redshift results for simulations of the CLASH photometry using the depths listed in Table 1 . From simul1 to simul4 we add complexity in galaxy physics such as extinction laws, emission lines and different galaxy libray as explained in Table 3, which increases the photo- $z$ scatter.

been adapted to reproduce COSMOS colours, number counts and redshift distribution as explained in Jouvel et al. (2009). The depth in each band is calculated to simulate the CLASH photometric depth shown in Table 1. The magnitude-error relation follows a Gaussian distribution based on galaxy fluxes and does not take galaxy sizes into account.

We produce four catalogues of increasing complexity in terms of galaxy physics. Table 3 summarizes the steps from simul1 (the simplest) to simul4 (most complex). Simul2 includes extinction as described by Calzetti et al. (2000) for the late type galaxies, and simul 3 also adds emission lines, using calibrations between the star formation rate and emission line fluxes (Kennicutt 1998). For simul1-3 we use the same libraries to create the mock catalogues and to calculate photo- $z$, i.e. the photo- $z$ library matches the truth. For simul4 we use the same library as simul 3 for the simulations, but the COSMOS library with extinction and emission lines to calculate photo- $z$.

Figure 10 shows the photo- $z$ results obtained from the four catalogues. Again, the scatter is given by the NMAD. The bottom left panel is the simplest case in which we reach a precision of $0.013(1+z)$. Adding extinction (simul2) and emission lines (simul3) decreases the precision slightly. Then, going to 
Table 4. Photoz results from the four simulations of the CLASH photometry.

\begin{tabular}{lcccc}
\hline \hline Simul & $\operatorname{Nmad}\left[z_{\mathrm{s}}<1\right]$ & $f_{\text {cata }}\left[z_{\mathrm{s}}<1\right]$ & $\operatorname{Nmad}\left[z_{\mathrm{s}}>1\right]$ & $f_{\text {cata }}\left[z_{\mathrm{s}}>1\right]$ \\
\hline Simul1 & 0.015 & $0.4 \%$ & 0.011 & $0.2 \%$ \\
Simul2 & 0.016 & $1.1 \%$ & 0.014 & $0.4 \%$ \\
Simul3 & 0.020 & $1 \%$ & 0.017 & $0.4 \%$ \\
Simul4 & 0.050 & $1.7 \%$ & 0.043 & $0.4 \%$ \\
\hline
\end{tabular}

Notes. We show the NMAD (scatter) for galaxies at $z_{\mathrm{s}}<1$ and $z_{\mathrm{s}}>1 . f_{\text {cata }}$ is the outlier fraction in $\%$ following the same definiton as $\eta, \eta$ being the number of outliers.

simul4 in the top right panel increases the scatter to $0.047(1+z)$. For simul4 we computed the systematic shifts to do a first order correction on the simulation and photo- $z$ library colour differences. In the best case, if the colours of our library are representative of observations, we should be close to simul3 with a scatter of $0.018 *(1+z)$. We note that in the magnitude range of the arcs sample $24<m(F 814 W)<26$, the NMAD in simul3 increase to $0.025(1+z)$. We do not expect the library colours to be fully representative of the variety we observe in the galaxy population. A precision of $3 \%$ to $4 \%(1+z)$ is within what we can expect from the CLASH observations.

Table 4 shows the photo- $z$ scatter and fraction of outliers for the four simulated cases at redshifts $z_{\mathrm{s}}<1$ and $z_{\mathrm{s}}>1$. Using the CLASH data, we reach a precision of $3.6 \%\left(1+z_{\mathrm{s}}\right)$ for galaxies at $z_{\mathrm{s}}<1$. This precision lies in between the $2 \%$ precision achieved with an optimal fully representative template library (simul3) and the 5\% precision achieved with a non-optimized library (simul4). At highredshift $z_{\mathrm{s}}>1$, the CLASH data yields a photo- $z$ precision of $2.6 \%\left(1+z_{\mathrm{s}}\right)$ and $3.1 \%\left(1+z_{\mathrm{s}}\right)$, respectively, with or without an $p d z_{-}$best cut. Simul3 and simul4 show an NMAD of $1.7 \%\left(1+z_{\mathrm{s}}\right)$ to $4.3 \%\left(1+z_{\mathrm{s}}\right)$. We conclude that the actual performance of the CLASH photo- $z$ are within expectations.

\section{Photometric redshifts of lensed galaxy images: MACS1206 example}

In the previous sections, we mainly focused on photo- $z$ for galaxies in the cluster field. In this section, we focus on the photo- $z$ quality of the "background lensed galaxies" (i.e., the arcs).

Strong lensing analysis incorporate the photo- $z$ estimates of individual galaxies in the lens modelling. Photo- $z$ scatter can have an impact on the strong lensing mass model. For example, erroneous photo- $z$ values can lead to model-tensions because the positions of lensed galaxies with inaccurate redshifts will not be correctly reproduced even if the model is largely based on arcs with reliable redshift values and the model is, thus, mostly reliable. We thus explore the degree to which the scatter in our photometric redshifts can bias the derived cluster mass profile parameters (e.g., central concentration). In this section, we focus, in particular, on the lensed background galaxies. The arcs that are projected near bright cluster galaxies may be particularly susceptible to higher photometric errors.

We study in detail the photo- $z$ measurements of fourteen lensed images, which have spec- $z$, behind the cluster MACS J1206.2-0847 ( $z=0.4385$; MACS1206 hereafter). This cluster was X-ray selected and its lensing properties were first studied in the Massive Cluster Survey, MACS (Ebeling et al. 2001, 2007, 2010). MACS1206 has high X-ray luminosity and a relatively smooth X-ray surface brightness profile which reflects the selection function of the MACS survey. MACS1206 first mass model was derived from F814W/HST imaging using

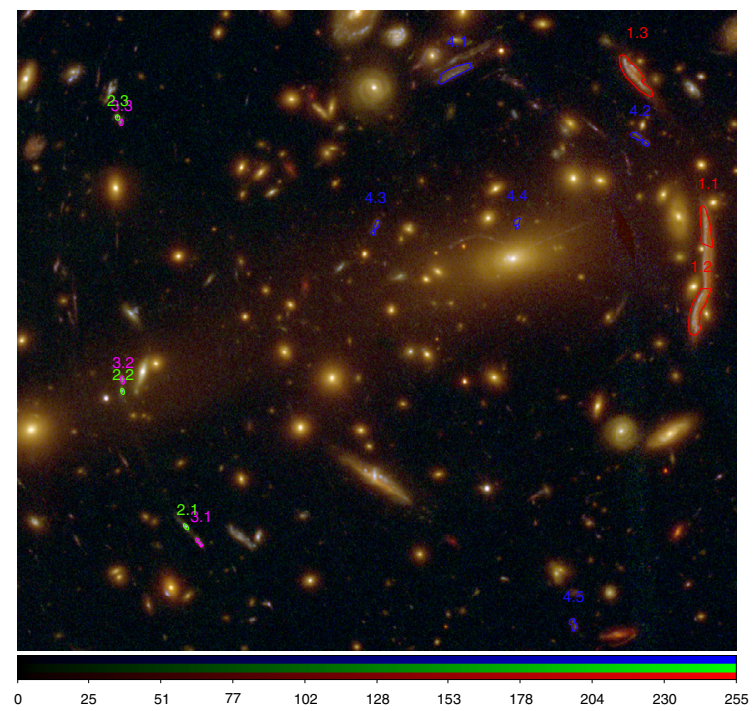

Fig. 11. Galaxy cluster MACS1206 at $z \sim 0.44$ in a combination of optical filters from the CLASH/HST observations (blue $=F 435 W+$ $F 475 W$, green $=F 606 W+F 625 W+F 775 W+F 814 W+F 850 L P$ red $=F 105 W+F 110 W+F 125 W+F 140 W+F 160 W)$. We show the strongly lensed multiple images described in Zitrin et al. (2012) for which we have spec-z. Systems 1-3 are highlighted in red, green, and blue, respectively. The image segmentation in these cases has been defined manually to optimize the reliability of the arc photometry. North is up, east is to the left. The pixel flux levels are given by the colour bar.

a single giant arc in Ebeling et al. (2009). Using the multi-band CLASH observations we have identified 47 new lensed images of 12 sources, four of which have spectroscopic redshifts (Zitrin et al. 2012) as shown in Fig. 11.

The first system is the one used in Ebeling et al. (2009) at a spectroscopic redshift of $z_{\mathrm{s}}=1.033$. The other systems were observed by the VIMOS/VLT Large Program 186.A-0798. Systems two and three are at $z_{\mathrm{s}}=3.03$ and system four at $z_{\mathrm{s}}=2.54$.

Below, we go through each system and present photo- $z_{\mathrm{s}}$ calculated with Le Phare and BPZ. We note that a first version of the CLASH photo- $z$ for the SL arcs of MACS1206 was presented in Zitrin et al. (2012). Zitrin et al. (2012) had not included the F336W data and presented photo- $z$ for the default SExtractor photometry. In this section, the photometry and photo- $z$ of the MACS1206 arcs are studied in greater detail with the SExtractor and tailored photometry. Arcs and multiple images can have very complicated shapes, and the standard SExtractor image detection and photometry may break the arcs into several parts, as shown by the segmentation map in Appendix C. This can affect the accuracy of the photometry for these objects. In the following, we compare Le Phare and BPZ results based on both custom manual photometry and on the standard SExtractor isophotal photometry for each arc. In Appendix $C$, we also show photo- $z$ results from BPZ using the isophotal photometry as well as the segmentation maps from SExtractor, cut-out images of the strong-lensing arcs, and the probability distributions and best-fit templates from BPZ.

Figure 12 shows, in the top row, the HST-image cutouts of some of the multiple image systems with the aperture that we used to derive the photometry of the different arcs. We note that each HST-image cutout shows several arcs from differents systems while the middle and bottom row show a different arcs system at each column. 

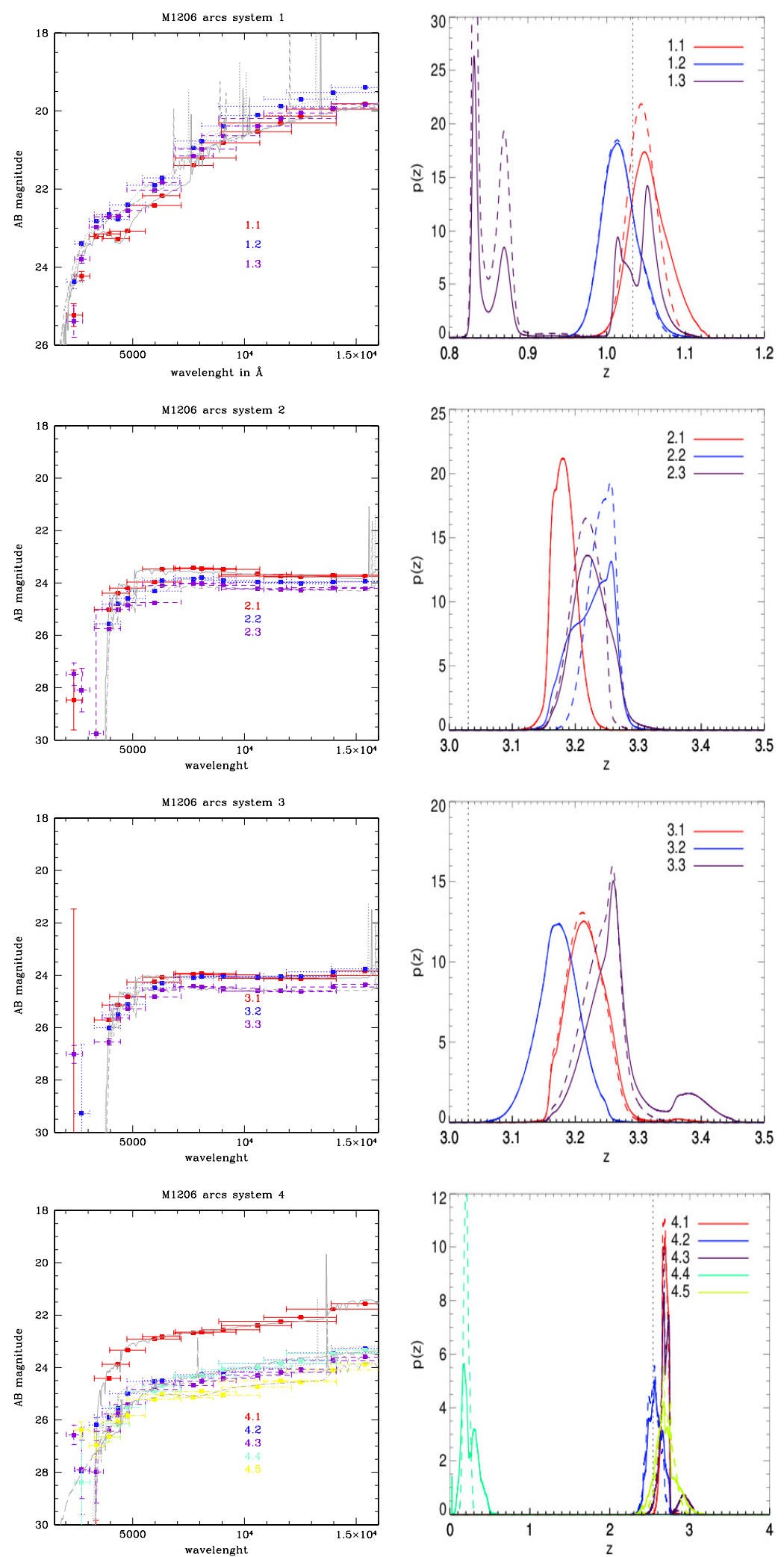

Fig. 12. Overview of the multiply imaged sources with spec- $z$ in MACS1206. The left panels show photo- $z$ best-fit templates of each strong-lensing image from Le Phare, and the CLASH/HST photometry. The vertical error bars are photometric errors and the horizontal are the width of the HST filters. In the right panels, the solid lines are the marginalized Le Phare $p(z)$ and the dashed lines are the $p(z)$ obtained by only considering the best-fit template in each case. We assume flat priors in redshift and template space.

For arc 1.1, custom photometry gives more accurate photo- $z$ results than the standard isophotal photometry with the difference between the spec- $z$ and the best-fit photometric redshift at less than $3 \%(1+z)$ for both BPZ and Le Phare. For arc 1.2, the custom aperture and the SExtractor-generated isophotal aperture are similar and the photo- $z$ for both apertures agrees with the spec- $z$ value to well within $3 \%(1+z)$. For arc 1.3 , the isophotal photometry gives more accurate photo- $z$ results than the custom photometry for both Le Phare and BPZ. The isophotal photometry draws a smaller aperture around arc 1.3 that yields higher precision colours which improves the photo- $z$ results.

System 1 shows good photo- $z$ results from both BPZ and Le Phare at less than $3 \%(1+z)$ with higher accuracy when using smaller aperture photometry, custom photometry for arc 1.1 and isophotal photometry for arc 1.3. All best-fits templates are starbursts galaxies. The lensed background galaxies are usually 
Table 5. Photo- $z$ for the multiple images with spec- $z$ in MACS1206, for the custom-tailored photometry and the standard isophotal photometry.

\begin{tabular}{|c|c|c|c|c|}
\hline & Arc & $\mathrm{LP} z_{\text {best }}-p d z_{\text {_b best }}$ & $\mathrm{BPZ} z_{\text {best }}-o d d s$ & $F 814 W$ \\
\hline Custom & 1.1 & $1.044_{-0.03}^{0.07}-100$ & $0.96_{-0.01}^{0.02}-98$ & 20.8 \\
\hline \multirow[t]{2}{*}{$z_{\mathrm{s}}=1.033$} & 1.2 & $1.01_{-0.04}^{0.05}-100$ & $0.98_{-0.02}^{0.01}-99$ & 20.4 \\
\hline & 1.3 & $0.83_{-0.01}^{0.24}-53$ & $0.977_{-0.07}^{0.02}-95$ & 20.6 \\
\hline \multirow[t]{3}{*}{ Isophotal } & 1.1 & $0.85_{-0.01}^{-0.01}-99$ & $0.74_{-0.07}^{0.07}-100$ & 20.6 \\
\hline & 1.2 & $1.07_{-0.03}^{0.02}-100$ & $1.04_{-0.08}^{0.08}-100$ & 21.0 \\
\hline & 1.3 & $1.05_{-0.02}^{0.03}-100$ & $1.05_{-0.08}^{0.08}-100$ & 22.4 \\
\hline \multirow{3}{*}{$\begin{array}{l}\text { Custom } \\
z_{\mathrm{s}}=3.03\end{array}$} & 2.1 & $3.18_{-0.03}^{0.04}-100$ & $3.367_{-0.004}^{0.011}-100$ & 23.5 \\
\hline & 2.2 & $3.25_{-0.10}^{0.02}-100$ & $3.39_{-0.02}^{0.01}-100$ & 23.9 \\
\hline & 2.3 & $3.22{ }_{-0.05}^{0.06}-100$ & $3.387_{-0.022}^{0.002}-100$ & 24.1 \\
\hline \multirow[t]{3}{*}{ Isophotal } & 2.1 & $3.21_{-0.07}^{0.05}-99$ & $0.41_{-0.06}^{0.06}-100$ & 22.9 \\
\hline & 2.2 & $3.20_{-0.09}^{0.20}-98$ & $0.41_{-0.06}^{0.06}-100$ & 22.9 \\
\hline & 2.3 & $3.64_{-0.06}^{0.08}-99$ & $3.68_{-0.18}^{0.18}-100$ & 23.6 \\
\hline \multirow{3}{*}{$\begin{array}{l}\text { Custom } \\
z_{\mathrm{s}}=3.03\end{array}$} & 3.1 & $3.21_{-0.05}^{0.06}-100$ & $3.367_{-0.044}^{0.003}-100$ & 24.0 \\
\hline & 3.2 & $3.17_{-0.06}^{0.07}-100$ & $3.25_{-0.02}^{0.03}-99$ & 24.0 \\
\hline & 3.3 & $3.26_{-0.08}^{0.14}-100$ & $3.39_{-0.03}^{0.02}-100$ & 24.5 \\
\hline \multirow[t]{3}{*}{ Isophotal } & 3.1 & $3.65_{-0.06}^{0.09}-99$ & $3.73_{-0.19}^{0.19}-100$ & 23.9 \\
\hline & 3.2 & $2.622_{-0.13}^{0.47}-94$ & $0.13_{-0.08}^{3.09}-83$ & 23.0 \\
\hline & 3.3 & $3.62_{-0.12}^{0.06}-95$ & $3.52_{-0.18}^{0.18}-98$ & 23.9 \\
\hline \multirow{5}{*}{$\begin{array}{l}\text { Custom } \\
z_{\mathrm{s}}=2.54\end{array}$} & 4.1 & $2.67_{-0.08}^{0.08}-100$ & $3.05_{-0.03}^{0.02}-100$ & 22.6 \\
\hline & 4.2 & $2.56_{-0.15}^{0.19}-100$ & $3.099_{-0.04}^{0.03}-96$ & 24.2 \\
\hline & 4.3 & $2.68_{-0.09}^{0.08}-100$ & $3.19_{-0.06}^{0.03}-93$ & 24.4 \\
\hline & 4.4 & $0.21_{-0.12}^{0.18}-78$ & $0.21_{-0.02}^{0.06}-49$ & 24.1 \\
\hline & 4.5 & $2.688_{-0.27}^{0.24}-99$ & $3.03_{-0.12}^{0.04}-73$ & 25.1 \\
\hline \multirow[t]{5}{*}{ Isophotal } & 4.1 & $2.54_{-0.08}^{0.07}-100$ & $2.99_{-0.16}^{0.16}-100$ & 22.2 \\
\hline & 4.2 & $2.35_{-0.19}^{-0.08}-72$ & $2.54_{-0.18}^{-0.10}-91$ & 24.1 \\
\hline & 4.3 & $1.93_{-0.02}^{0.29}-61$ & $2.35_{-0.19}^{0.19}-85$ & 24.3 \\
\hline & 4.4 & $0.520_{0.000}^{0.005}-145$ & $0.36_{-0.05}^{0.07}-97$ & 20.6 \\
\hline & 4.5 & $2.67_{-0.21}^{0.60}-65$ & $3.04_{-0.21}^{0.16}-92$ & 24.7 \\
\hline
\end{tabular}

very blue young galaxies and are thus best-fitted by starburst templates. One would however need an individual template fitting procedure to determine the type of each of these galaxies.

System 2 has three arcs from a source galaxy at redshift 3.03 for which spectroscopy has been obtained from the on going VLT campaign. We have good photometry in the visible and NIR for all the arcs.

The photo- $z$ of the three images of system 2 have a $2 \sigma$ confidence region that is off by 0.15 in redshift. The Balmer break being out of the CLASH filter range, the Lyman break is the only strong colour that will help the fitting as shown in Fig. 12. The custom photometry shows better photo- $z$ results than the isophotal for all arcs with more consistent photo- $z$ values closer to the spectroscopic redshift even if off by at least 0.15 in redshift.

As for system 2, we do not find a lot of flux in the UVIS filters. The photometric redshift of system 3 is very similar to system 2 and the images are radially very close to each other. Systems 2 and 3 are probably a galaxy group lensed by MACS1206.

Arcs of system 3 have very similar photometry except in the bluest filter. They show similar photo- $z$ fit as you can see in Fig. 12 and Table 5. The custom photometry gives photo- $z$ estimates closer to the spec- $z$ than the isophotal photometry even if still off by at least 0.2 in redshift. The standard photometry shows photo- $z$ results that are off by 0.5 in redshift and values that are not always consistent for all the arcs. BPZ and Le Phare show similar results for both photometry.

Arcs of system 4, as for systems 2 and 3, do not have much flux in the UVIS filters. However, the Balmer break lies in the NIR filters and improves the reliability of the high-redshift solution for arcs 4.1, 4.2, 4.3 and 4.5. Arc 4.4 is very close to the MACS1206 BCG and is likely contaminated by the BCG light as illustrated in the bottom panel right figure of Fig. 12. This issue will be adressed in a further paper (Molino et al., in prep.). The photo- $z$ of arc 4.1, 4.2 and 4.5 are at less than $2 \sigma$ from the spectroscopic redshift and at less than $3 \%(1+z)$. All images except 4.4 have good fits and are very close to the spectrocopic redshift, for Le Phare using the custom photometry. BPZ shows slightly biased high photometric redshift for the custom photometry.

For all the systems, BPZ and Le Phare show very close results for most cases and at less than $2 \%(1+z)$. Both BPZ and Le Phare give consistent and better results using the custom photometry compared to the standard isophotal photometry since we usually draw smaller tailored apertures compared to the standard one. Smaller aperture photometry shows higher colour accuracy and better photo- $z$ from Le Phare and BPZ for all arcs.

\section{MACS1206 photometric redshift catalogue}

We release a photo- $z$ catalogue of all sources in MACS1206 CLASH/HST field. This catalogue includes the multi-band photometry information (including upper-limits) and BPZ photo- $z$ for each object that has been detected as described in Sect. 2. This catalogue is currently online in the Mikulski Archive for Space Telescopes (MAST) system ${ }^{8}$. To the online MAST catalogue, we add our new Le Phare photo- $z$.

There are a total of 3510 objects in the catalogue, in an area of $4.08 \mathrm{arcmin}^{2}$ covered by the CLASH programme. We give the coordinates of each objects along with other SExtractor output such as an estimation of the size given by the full-width-halfmaximum, the area used to derive the photometry, an estimate of the ellipticity calculated from the second moment of the light distribution (Bertin \& Arnouts 1996) and a probability of the object being a star called stellarity, determined by a neural network approach. For the photo- $z$ results, we give the best-fit redshift with a 68 and $95 \%$ confidence region for both BPZ and Le Phare. We also include the odds $-p d z$ _best values in each case.

Figure 13 shows Le Phare and BPZ photo- $z$ in the MACS1206 field for galaxies with $S / N>10$ in $F 814 W$ and a stellarity lower than 0.08 . Stellarity is close to 0 for galaxies and goes up to 1 for stars. In MACS1206 case, a stellarity lower than 0.08 select $86 \%$ of the $10 \sigma F 775 \mathrm{~W}$ detected sample. Le Phare and BPZ agree very well. There are discrepancies for some galaxies placed at low redshift by Le Phare but at high redshift for BPZ. These discrepancies represent $7 \%$ of the galaxies with $S / N>10$ and happen where we usually find catastrophic outliers due to the confusion between the Lyman and Balmer breaks. Those galaxies will likely yield poor photometric redshifts although no definitive conclusion can be drawn without the spec- $z$ information. Most of the galaxies for which BPZ and Le Phare disagree have $p d z \_$best lower than 0.4 , and so they can be pruned with an $p d z \_$best cut.

Figure 14 shows different selections of galaxies to have some understanding of the photo- $z$ quality in comparing Le Phare and BPZ. The sample UVIS+ACS+NIR and ACS+NIR is made

8 http://archive.stsci.edu/prepds/clash/ 


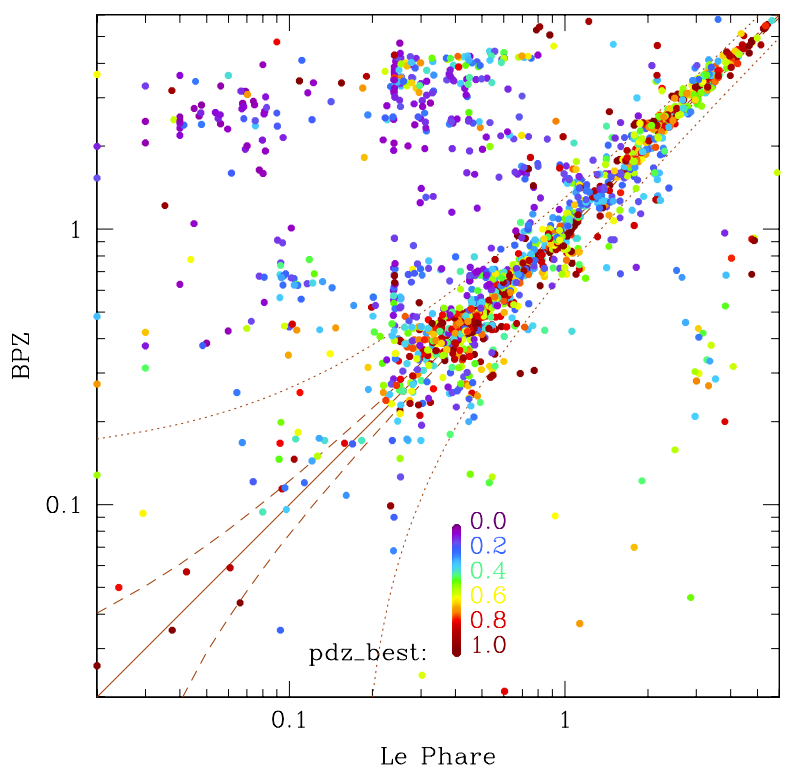

Fig. 13. Comparison between Le Phare and BPZ photo- $z$ in MACS1206 field for galaxies with $S / N>10$ in $F 814 W$ and stellarity lower than 0.08. Colour scale with the pdz_best parameter, taken from Le Phare. The dashed and dotted lines represent respectively $2 \%(1+z)$ and $15 \%(1+z)$. Le Phare and BPZ agrees well except a small fraction of faint galaxies with low confidence photo- $z$ that Le Phare places at low redshift and BPZ spreads over the redshift range.

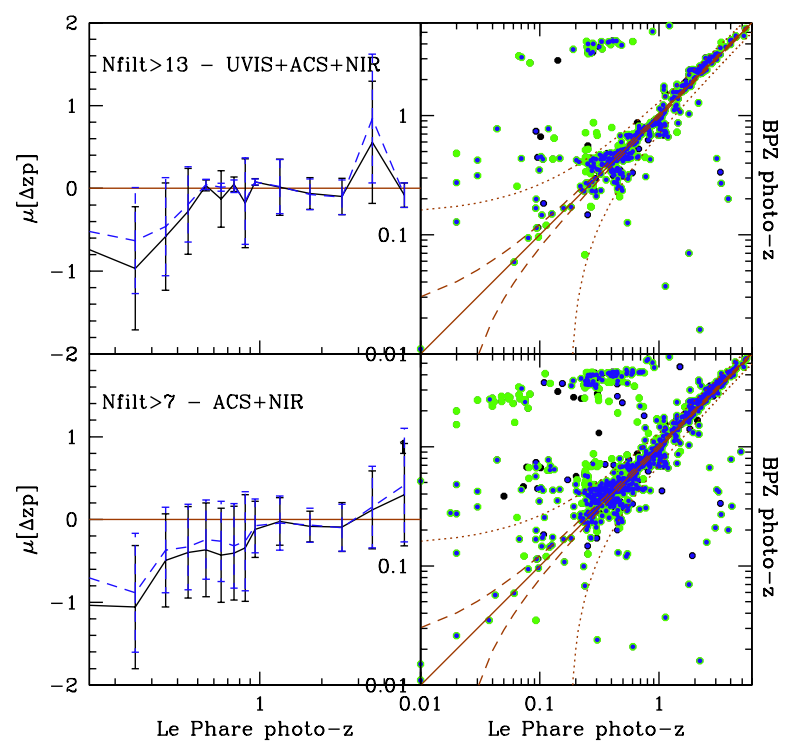

Fig. 14. Comparison between Le Phare and BPZ photo- $z$ in MACS1206 field for galaxies with $S / N>10$ in $F 814 W$ and stellarity lower than 0.08. Figures on the left show the mean value of the $\Delta z_{\mathrm{p}}=z_{\mathrm{p}}^{\mathrm{Le}}$ Phare $z_{\mathrm{p}}^{\mathrm{BPZ}}$ as a function of $z_{\mathrm{p}}^{\mathrm{Le} \text { Phare }}$ for ACS +NIR and UVIS + ACS +NIR photometry respectively bottom and top panels. The blue and black lines are respectively Le Phare and BPZ photo- $z$. The error bars are the rms between Le Phare and BPZ photo-z. Right panels show the Le Phare vs. BPZ photo- $z$. The green and blue dots show a selection of respectively BPZ $\chi^{2}$ and Le Phare $p d z \_$best $85 \%$ highest confidence redshifts. The dashed and dotted lines represent respectively $2 \%(1+z)$ and $15 \%(1+z)$.

from galaxies with at least respectively 13 and 7 filters detection. From the number of filter selection, we conclude that galaxies with a high number of detections are likely to have a good photo- $z$ estimates. We also do a pre-selection of the highest confidence $85 \%$ galaxies from BPZ $\chi^{2}$ and Le Phare $p d z \_b e s t$ and

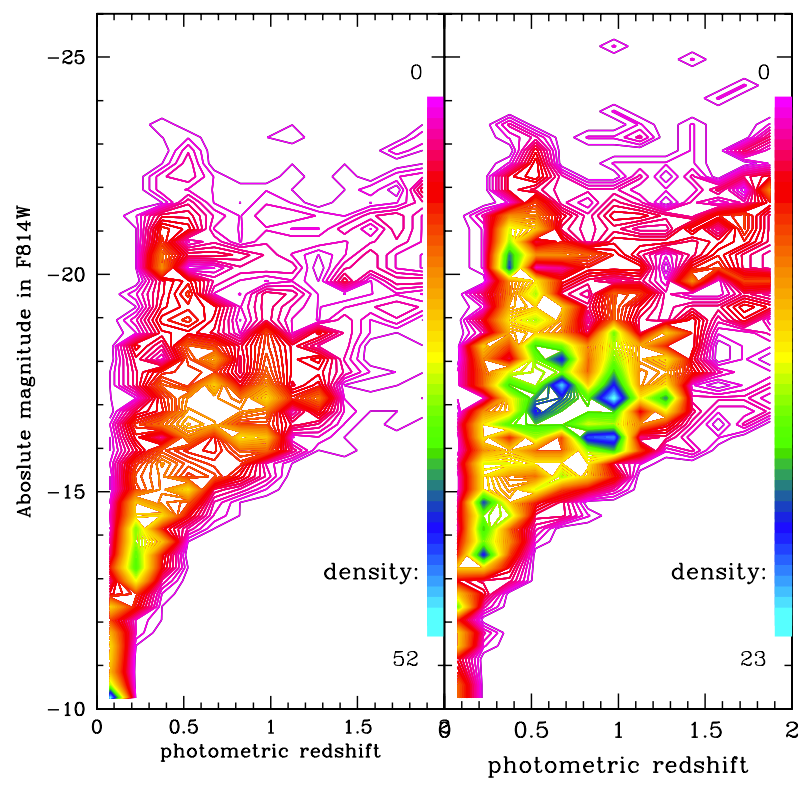

Fig. 15. Contour showing the galaxy number density as a function of the absolute magnitude in $F 814 \mathrm{~W}$ and redshift for MACS1206 galaxy cluster. The absolute magnitude is derived from Le Phare photo- $z$ fitting. The left and right panels have respectively no pdz_best cut and a cut at 0.1 . The galaxy number density is in linear colour scale. The low redshift and intrisically faint galaxies show a poor photo- $z$ estimation with $p d z \_$best $<0.1$.

eliminate most of the BPZ-Le Phare outliers. The corresponding values of $\left(\chi^{2}, p d z \_b e s t\right)$ are $(1.44,37)$. For more details about the performance of these selections, see Fig. B.2 which shows the selections as a function of magnitude and their completness as a function or magnitude and redshift.

Figure 15 shows the galaxy number density for MACS1206 galaxy cluster. The left panel of Fig. 15 shows a high density of faint galaxies at low redshift. On the right panel of Fig. 15, we show the same absolute magnitude-redshift-number density relation with a photo- $z$ quality cut of $p d z \_b e s t>0.1$. The quality cut removes most of the faint low-redshift galaxies. The faint low-redshift galaxies are just an artefact from a bad fitting due to faint poorly constrained photometry.

Figure 16 shows photo- $z$ histograms of galaxies with a $S / N>10$ in $F 814 W$ of Le Phare in black solid line and BPZ in blue dashed line. The cyan solid curve is at MACS1206 cluster redshift. We remove the stars using SExtractor stellarity flag. The main figure shows BPZ and Le Phare photo- $z$ with the signal-to-noise and stellarity selection. The top right corner histograms show BPZ and Le Phare photo- $z$ with a quality cut which selects $85 \%$ of the highest confidence photo- $z$ of the galaxy sample ( $p d z \_$best, odds $\left.>0.15,0.31\right)$ for the top right corner histograms. The bottom panel of Fig. 16 shows ratio of Le Phare and BPZ histograms. BPZ and Le phare agree well from $z_{\mathrm{p}}>0.2$ up to redshift $z_{\mathrm{p}}<2$. At $z_{\mathrm{p}}>2 \mathrm{BPZ}$ finds more galaxies than Le Phare. As described in the above paragraph, we note that Le Phare has an unrealistic number of galaxies at low redshift $z_{\mathrm{p}}<0.2$ that are placed at high redshift by BPZ that can be pruned with an odds cut.

Figure 17 shows the mean photo- $z$ as a function of clustercentric radius of galaxies located behind MACS1206 with a $S / N>10$ in $F 775 \mathrm{~W}$, stellarity lower than 0.08 . It shows a mean redshift higher close to the centre of the cluster due to the greater magnification near the cluster centre. In the radial bin closest to the cluster centre, the mean redshift is around 2.5, while for the 


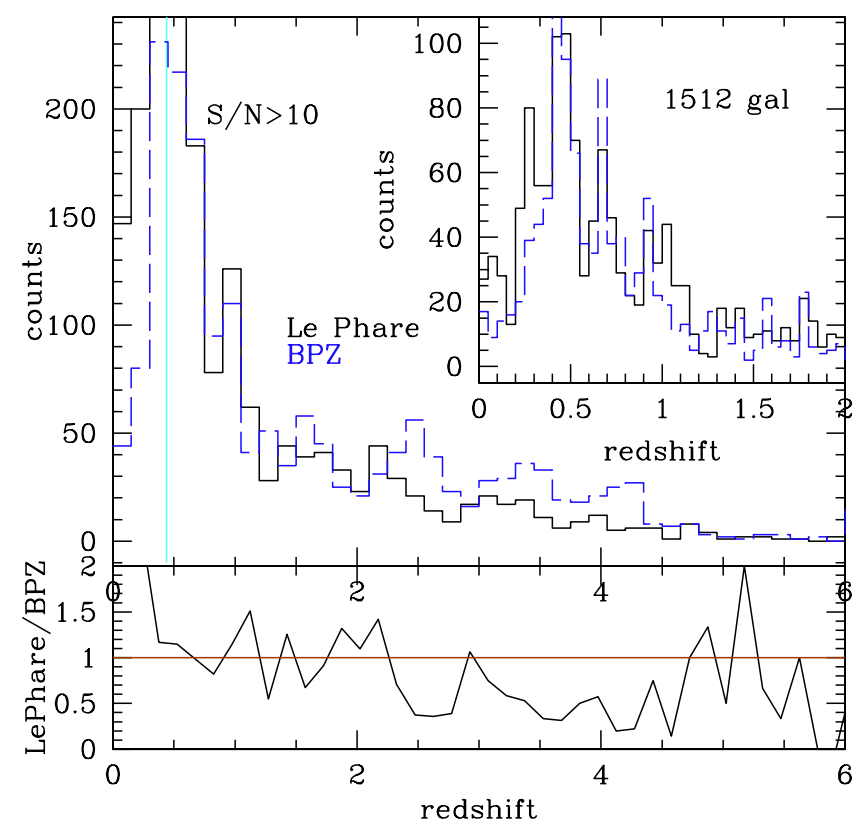

Fig. 16. Photometric redshift histogram of MACS1206 using BPZ in dashed blue line and Le Phare in solid black line. The solid cyan line is MACS1206 redshift at $z \sim 0.44$. BPZ and Le Phare have similar redshift distribution and show a well defined peak at the cluster location. The top right corner panel displays BPZ and Le Phare photo- $z$ histograms for a selection of $o d d s$ and $p d z$ best values in order to select the best $85 \%$ of galaxies for both BPZ and Le Phare.

radial bins further away the mean redshift is around 2 and does not change much as we go further away from the cluster centre.

\section{Sensibility of the mass reconstruction from SL to the photo-z accuracy}

Now we consider the impact of the photo- $z$ uncertainty on the lens modelling. The accuracy of the photo- $z_{\mathrm{s}}$ measured for arcs and lensed images directly influences the accuracy of the mass model through the lens equation, which connects the unknown source position $\beta$ with the image position $\theta$

$\beta=\theta-\frac{D_{\mathrm{ls}}}{D_{\mathrm{s}}} \hat{\alpha}(\theta)$

The mass model determines the deflection field $\hat{\alpha}$ and the photo- $z$ enters in the distance ratio $D_{\mathrm{ls}} / D_{\mathrm{s}}$. Figure 18 shows how the relative photo- $z$ accuracy is mapped to the distance ratio using standard linear error propagation. It is clear that low- redshift sources are the most challenging. The relative accuracy of the distance ratio can be regarded as the relative error on the overall mass scale of the lens since $\hat{\alpha} \propto M$ so, e.g., a $z=2$ source lensed by a $z=0.3$ cluster will give rise to a relative uncertainty on $D_{\mathrm{ls}} / D_{\mathrm{s}}$ and on the total mass of $\sim 1 \%$ if the photo- $z$ uncertainty is $4 \%$. However, the exact influence of the photo- $z$ accuracy on the lens model will depend on the number, positions, and redshifts of the lensed images in a complex way, and it may also depend on whether the lens model is parametric or not. This will be assessed on a case-by-case basis.

However, we can also regard the photo- $z$ uncertainty as a positional uncertainty in the image plane, $\delta \alpha_{z}$, through the lens equation. The relative accuracy of the distance ratio in Fig. 18 is then equal to the relative accuracy on the distance between the image and the model-predicted source position. This approach

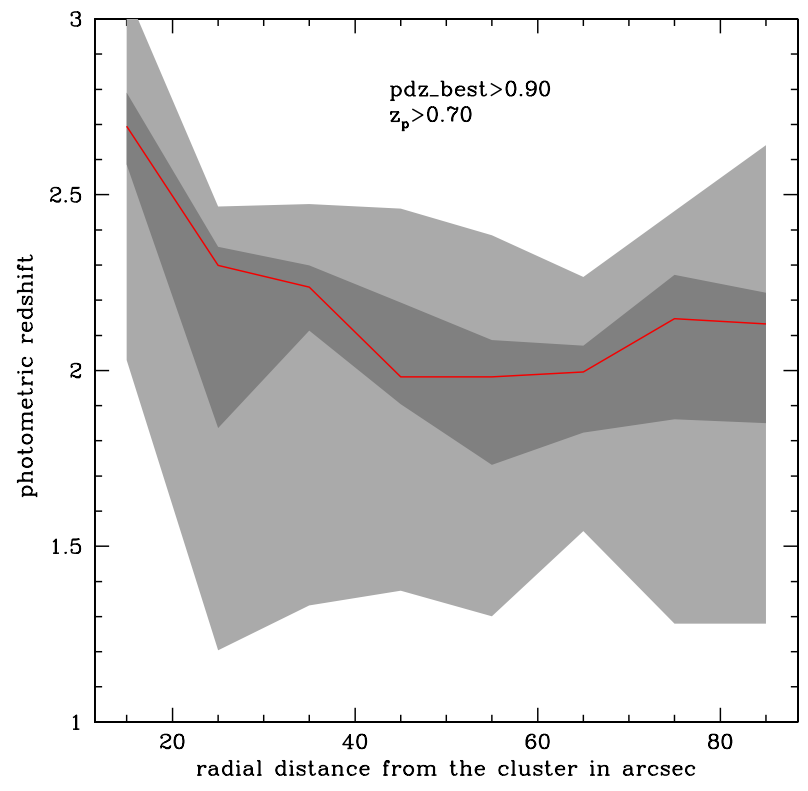

Fig. 17. Mean photo- $z$ of galaxies located behind MACS1206 in solid red line as a function of angular radial distance from the cluster centre. The dark grey area reprensents the mean $68 \%$ area around the mean and the light grey the $95 \%$ area. Photo- $z$ are derived from Le Phare code.

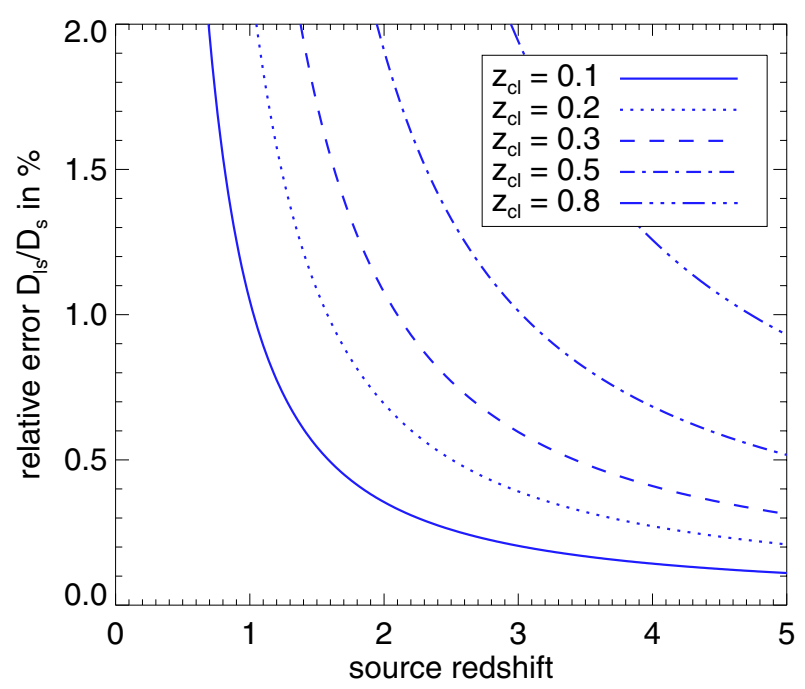

Fig. 18. Relative error on the distance ratio $D_{\mathrm{ls}} / D_{\mathrm{s}}$ as a function of source redshift $z_{\mathrm{s}}$ for various lens redshifts $z_{\mathrm{cl}}$, assuming a photo-z precision of $4 \%$.

facilitates comparison with other positional uncertainties that affect the lensing analysis. To be specific, we base our comparison on the 168 lensed images produced by Abell 1689 and their corresponding source positions as found using the lens model of Coe et al. (2010). We imagine that each image has a photo- $z$ and that the overall photo- $z$ precision is $4 \%$. The disttribution of the photo- $z$ positional uncertainties, which we label $\delta \alpha_{z}$ are shown in Fig. 19. We take this distribution as a reasonable sample estimate of the impact of photo- $z$ accuracy.

For HST imaging, the accuracy of the astrometric solution is similar to the pixel scale, 0.05 arcsec for the ACS/HST camera. It is clear from Fig. 19 that a $4 \%$ photo- $z$ precision implies that, for most images, $\delta \alpha_{z}$ will be larger than the astrometric error. However, larger positional errors are predicted: Jullo et al. (2010) estimated that images would pick up additional deflections of more than 1 arcsec from lensing by the large-scale 


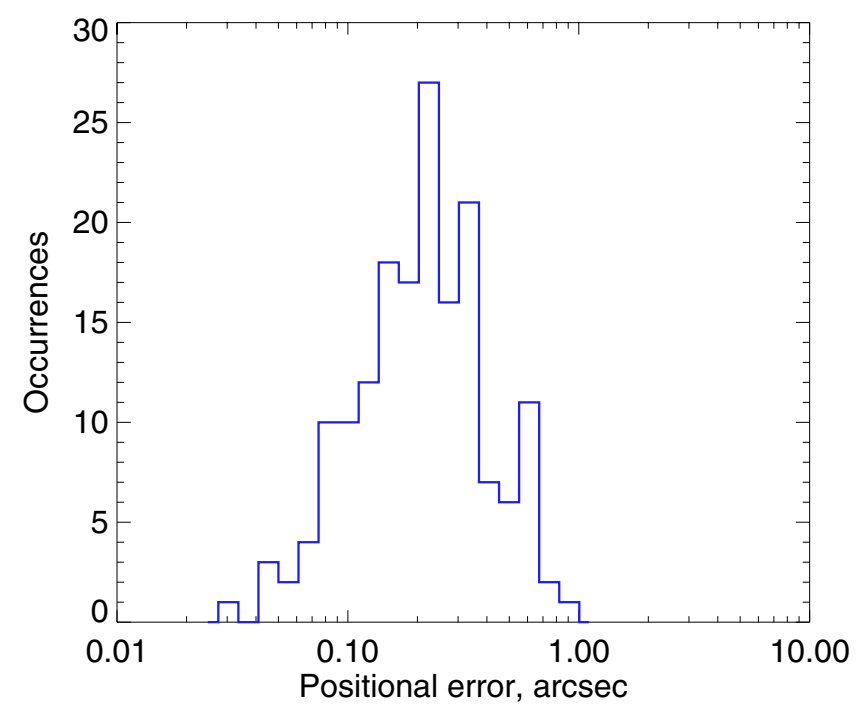

Fig. 19. Distribution of photo- $z$ positional errors for the 168 images lensed by Abell 1689 .

structure along the line-of-sight, and it is clear from Fig. 19 that we expect almost all $\delta \alpha_{z}$ to be smaller than this. In a more detailed matter power spectrum analysis, Host (2012) showed that the impact of large-scale structure increases with redshift and distance on the sky to the cluster centre, which means that lowredshift arcs will be relatively more affected by the photo- $z$ accuracy. We calculate the deflection $\delta \alpha_{\text {LSS }}$ using the prescription of Host (2012), and we find that $\delta \alpha_{z}>\delta \alpha_{\text {LSS }}$ for $6 \%$ of the Abell 1689 images if the photo- $z$ precision is $4 \%$ while this happens for only one image if the precision is $2 \%$. Hence, we conclude that the impact of the photo- $z$ accuracy of the mass model is most likely subdominant in comparison to the uncertainty associated with lensing by large-scale structure along the line of sight if we can achieve a photo- $z$ precision of $4 \%$ or better. We note that this model is valid when the lensed images do not sit on a strong-lensing critical line. In this last case, the non-linearity of equations makes any predictions difficult to realize.

Finally, there is the possibilty of catastrophic errors arising from the Lyman-Balmer break degeneracy, which can give rise to two solutions in the photo- $z$ fitting. However, this is usually a benign case for the purpose fitting the lens model. It is extremely unlikely that the low- $z$ solution is a viable redshift for a lensed image, in fact, for CLASH clusters, it may well be a lower redshift than the cluster lens itself. Even if the low- redshift solution is viable for an arc it will be difficult to fit the observed image positions since the separation between the lensed images would not accommodate the low predicted $D_{\mathrm{ls}} / D_{\mathrm{s}}$ - as long as there are other lensed images constraining the mass model. Hence, catastrophic outliers will mostly be an issue for the identification of lensed images in the first place, and in that case there are other constraints such as morphology which can be used.

\section{Conclusion}

In this study, we evaluated the photo- $z$ accuracy using 16 bands HST going from UV to NIR for 17 out of the $25 \mathrm{CLASH}$ clusters. We compare two photo- $z$ methods, BPZ and Le Phare for all the galaxies in 17 cluster fields and test them against spectrocopic redshifts. We studied separately the lensed background galaxies with a sample of 63 galaxies from the cluster member and foreground galaxies with 327 galaxies. Our main conclusions are:

- Le Phare photo- $z$ for the lensed background galaxies are somewhat more accurate than those for the foreground and cluster members when adding zeropoint adjustements.

- Using Le Phare, we reach a precision of $3 \%(1+z)$ for the lensed background galaxies, which is reduced to $2.4 \%(1+z)$ after removing outliers (based on the quality of the fit). Both BPZ and Le Phare have similar photo- $z$ estimation for galaxies in the foreground and cluster field with a correponding values of $4.0 \%(1+z)$ and $3 \%(1+z)$. This precision for the arcs satisfies the requirement for the mass model reconstruction as shown in Sect. 7. Using a simple error propagation going from the photo- $z$ to the lens model, we find a $1 \%$ error on the derived lens model using a photo- $z$ scatter of $4 \%(1+z)$ for a cluster at $z=0.3$ and a source at $z=2$. This is however a simplistic model and a case by case study for each cluster is necessary for more detail.

- The $p(z)$-based parameters odds and pdz_best are demonstrated to be useful estimators of the quality of the derived photometric redshifts.

- Isophotal photometry, in general, performs better than other types of photometry although similar results are achieved with 20 pixels aperture photometry.

- We verified the above conclusions using mock galaxy catalogues that included varying degrees of complexity in the underlying galaxy physics.

- For MACS1206 we compare photo- $z$ for the arcs using 2 photometric measurements and find that using Le Phare and BPZ photo- $z$ have a precision higher than $3 \%(1+z)$. Smaller aperture photometry gives better photo- $z$ results usually obtained from the tailored aperture photometry. Photo- $z$ results for the lensed background galaxies with the default SExtractor photometry show a scatter of $5 \%(1+z)$ demonstrating the utility of the tailored photometry. For MACS1206 lensed systems 1, 2 and 4, Le Phare and $\mathrm{BPZ}$ agrees within $2 \sigma$ in redshift for the arcs and for the field galaxies

We are also working on improving the photometry with a background substraction model adjusted for each cluster using shapelets (Molino et al., in prep.).

Acknowledgements. S.J. and O.H. acknowledge STFC-supported Post-doctoral Felowships at UCL, O.L. acknowledges a Royal Society Wolfson Research Merit Award, a Leverhulme Senior Research Fellowship and an Advanced ERC Grant. S.J. is supported by the Spanish Science MinistryAYA2009-13936 Consolider-Ingenio CSD2007-00060, project2009SGR1398 from Generalitat de Catalunya and by the European Commissions Marie Curie Initial Training Network CosmoComp (PITN- GA-2009-238356). The Dark Cosmology Centre is funded by the Danish National Research Foundation. S. Seitz acknowledges support from the Transregional Collaborative Research Centre TRR 33 "The Dark Universe" and from the DFG cluster of excellence "Origin and Structure of the Universe". This research is partially supported by PRIN INAF 2010: "Architecture and Tomography of Galaxy Clusters". A.F. acknowledges support from INAF through PRIN 2008 and 2010 grants. Support for A.Z. is provided by NASA through Hubble Fellowship grant \#HST-HF-51334.01-A awarded by STScI. Part of this work was also supported by contract research "Internationale Spitzenforschung II/2-6" of the Baden Württemberg Stiftung. A.M. acknowledges support from AYA2006-14056BES-2007-16280.

\section{References}

Abazajian, K. N., Adelman-McCarthy, J. K., Agüeros, M. A., et al. 2009, ApJS, 182,543

Benítez, N. 2000, ApJ, 536, 571

Benítez, N., Ford, H., Bouwens, R., et al. 2004, ApJS, 150, 1 
Bertin, E., \& Arnouts, S. 1996, A\&AS, 117, 393

Biviano, A., Rosati, P., Balestra, I., et al. 2013, A\&A, 558, A1 Bolzonella, M., Miralles, J.-M., \& Pelló, R. 2000, A\&A, 363, 476 Brammer, G. B., van Dokkum, P. G., \& Coppi, P. 2008, ApJ, 686, 1503 Bruzual, G., \& Charlot, S. 2003, MNRAS, 344, 1000

Calzetti, D., Armus, L., Bohlin, R. C., et al. 2000, ApJ, 533, 682

Coe, D., Benítez, N., Sánchez, S. F., et al. 2006, AJ, 132, 926

Coe, D., Benítez, N., Broadhurst, T., \& Moustakas, L. A. 2010, ApJ, 723, 1678

Coe, D., Umetsu, K., Zitrin, A., et al. 2012, ApJ, 757, 22

Cohen, J. G., \& Kneib, J.-P. 2002, ApJ, 573, 524

Coleman, G. D., Wu, C.-C., \& Weedman, D. W. 1980, ApJS, 43, 393

Collister, A. A., \& Lahav, O. 2004, PASP, 116, 345

Csabai, I., Dobos, L., Trencséni, M., et al. 2007, Astron. Nachr., 328, 852

Dahlen, T., Mobasher, B., Somerville, R. S., et al. 2005, ApJ, 631, 126

Ebeling, H., Edge, A. C., \& Henry, J. P. 2001, ApJ, 553, 668

Ebeling, H., Barrett, E., Donovan, D., et al. 2007, ApJ, 661, L33

Ebeling, H., Ma, C. J., Kneib, J.-P., et al. 2009, MNRAS, 395, 1213

Ebeling, H., Edge, A. C., Mantz, A., et al. 2010, MNRAS, 407, 83

Fabricant, D., Fata, R., Roll, J., et al. 2005, PASP, 117, 1411

Fioc, M., \& Rocca-Volmerange, B. 1997, A\&A, 326, 950

Gerdes, D. W., Sypniewski, A. J., McKay, T. A., et al. 2010, ApJ, 715, 823

Giavalisco, M., Ferguson, H. C., Koekemoer, A. M., et al. 2004, ApJ, 600, L93

Greisel, N., Seitz, S., Drory, N., et al. 2013, ApJ, 768, 117

Guennou, L., Adami, C., Ulmer, M. P., et al. 2010, A\&A, 523, A21

Guzzo, L., Schuecker, P., Böhringer, H., et al. 2009, A\&A, 499, 357

Halkola, A., Hildebrandt, H., Schrabback, T., et al. 2008, A\&A, 481, 65

Hildebrandt, H., Arnouts, S., Capak, P., et al. 2010, A\&A, 523, A31

Hoaglin, D. C., Mosteller, F., \& Tukey, J. W. 1983, Understanding robust and exploratory data analysis (New York: Wiley)

Host, O. 2012, MNRAS, 420, L18

Ilbert, O., Arnouts, S., McCracken, H. J., et al. 2006, A\&A, 457, 841

Ilbert, O., Capak, P., Salvato, M., et al. 2009, ApJ, 690, 1236

Jones, D. H., Saunders, W., Colless, M., et al. 2004, MNRAS, 355, 747

Jouvel, S., Kneib, J.-P., Ilbert, O., et al. 2009, A\&A, 504, 359

Jullo, E., Natarajan, P., Kneib, J.-P., et al. 2010, Science, 329, 924

Kennicutt, Jr., R. C. 1998, ARA\&A, 36, 189

Koekemoer, A. M., Faber, S. M., Ferguson, H. C., et al. 2011, ApJS, 197, 36

Kron, R. G. 1980, ApJS, 43, 305

Lamareille, F., Contini, T., Le Borgne, J.-F., et al. 2006, A\&A, 448, 893

Lemze, D., Postman, M., Genel, S., et al. 2013, ApJ, 776, 91

Maturi, M., Mizera, S., \& Seidel, G. 2013, A\&A, submitted [arXiv: 1305.3608]

Newman, A. B., Treu, T., Ellis, R. S., \& Sand, D. J. 2011, ApJ, 728, L39

Pelló, R., Rudnick, G., De Lucia, G., et al. 2009, A\&A, 508, 1173

Polletta, M., Tajer, M., Maraschi, L., et al. 2007, ApJ, 663, 81

Postman, M., Coe, D., Benítez, N., et al. 2012, ApJS, 199, 25

Richard, J., Kneib, J.-P., Ebeling, H., et al. 2011, MNRAS, 414, L31

Rines, K., Geller, M. J., Diaferio, A., \& Kurtz, M. J. 2013, ApJ, 767, 15

Sand, D. J., Treu, T., Ellis, R. S., Smith, G. P., \& Kneib, J.-P. 2008, ApJ, 674, 711

Schlegel, D. J., Finkbeiner, D. P., \& Davis, M. 1998, ApJ, 500, 525

Scoville, N., Aussel, H., Brusa, M., et al. 2007, ApJS, 172, 1

Smith, G. P., Kneib, J.-P., Smail, I., et al. 2005, MNRAS, 359, 417

Stern, D., Jimenez, R., Verde, L., Stanford, S. A., \& Kamionkowski, M. 2010, ApJS, 188, 280

Umetsu, K., Medezinski, E., Nonino, M., et al. 2012, ApJ, 755, 56

Vanzella, E., Cristiani, S., Fontana, A., et al. 2004, A\&A, 423, 761

White, S. D. M., Clowe, D. I., Simard, L., et al. 2005, A\&A, 444, 365
Wuyts, S., Labbé, I., Schreiber, N. M. F., et al. 2008, ApJ, 682, 985

Zitrin, A., Broadhurst, T., Coe, D., et al. 2011, ApJ, 742, 117

Zitrin, A., Rosati, P., Nonino, M., et al. 2012, ApJ, 749, 97

1 Institut de Ciències de l'Espai (IEEC-CSIC), 08193 Bellaterra (Barcelona), Spain

e-mail: jouvel@ice.cat

2 University College London, Gower street, London WC1E 6BT, UK

3 Dark Cosmology Centre, Niels Bohr Institute, University of Copenhagen, Juliane Maries Vej 30, 2100 Copenhagen, Denmark

4 Space Telescope Science Institute, 3700 San Martin Drive, Baltimore MD 21218, USA

5 European Southern Observatory (ESO), 85748 Garching, Germany

6 Institut für Astronomie und Astrophysik, Universitäts-Sternwarte München, 81679 München, Germany

7 Department of Theoretical Physics, University of the Basque Country UPV/EHU, 48080 Bilbao, Spain

8 Ikerbasque, Basque Foundation for Science, 48011 Bilbao, Spain

9 Instituto de Astrofísica de Andalucía (IAA-CSIC), 18008 Granada, Spain

10 Department of Physics and Astronomy, The Johns Hopkins University, Baltimore, MD, USA

11 Institut für Theoretische Astrophysik, Zentrum für Astronomie, Institut für Theoretische Astrophysik, Albert-Ueberle-Str. 2, 29120 Heidelberg, Germany

12 Leiden Observatory, Leiden University, 2333 Leiden, The Netherlands

13 Department of Physics and Astronomy, Michigan State University, East Lansing, MI, USA

14 Department of Astronomy, University of California, Berkeley CA 94720, USA

15 Instituto de Astrofísica y AIUC, P. Universidad Católica de Chile, 306 Casilla, Santiago 22, Chile

16 Carnegie Observatories, Carnegie Institute for Science, Pasadena, CA, USA

17 Center for Cosmology and Astro-Particle Physics, The Ohio State University, 191 W. Woodruff Ave., Columbus OH 43210, USA

18 INAF - Osservatorio Astronomico di Bologna, INFN, Sezione di Bologna, 40127 Bologna, Italy

19 Jet Propulsion Laboratory, California Institute of Technology, 4800 Oak Grove Dr, Pasadena, CA 91109, USA

20 Institute of Astronomy and Astrophysics, Academia Sinica, PO Box 23-141, 10617 Taipei, Taiwan

21 INAF - Osservatorio Astronomico di Capodimonte, via Moiariello 16, 80131 Napoli, Italy

22 Department of Physics and Astronomy, Siena College, 515 Loudon Road, Loudonville NY 12211, USA

23 INAF - Istituto di Astrofisica Spaziale e Fisica cosmica (IASF) Milano, via Bassini 15, 20133 Milano, Italy

24 Cahill Center for Astronomy and Astrophysics, California Institute of Technology, MS 249-17, Pasadena CA 91125, USA

25 INAF - Osservatorio Astronomico di Trieste, via G.B. Tiepolo 11, 40131 Trieste, Italy

26 Hubble Fellow 


\section{Appendix A: Spectroscopic redshifts and systematic shifts}

Table A.1 gives the number of very secure spectroscopic redshift available for galaxies that lie within the CLASH/HST fields of view. Most spec-z's have been targeted by the VIMOS/VLT Large Program 186.A-0798 (Rosati, in prep.). Other spec- $z$ come from GISMO observations on Magellan telescopes, VLT observations (Lamareille et al. 2006), the 6DF survey (Jones et al. 2004), the SDSS DR7 (Abazajian et al. 2009), the MMT/Hectospec survey (Fabricant et al. 2005; Rines et al. 2013) and archival data from Ebeling et al. (2009); Sand et al. (2008); Smith et al. (2005); Newman et al. (2011); Richard et al. (2011); Guzzo et al. (2009); Halkola et al. (2008); Cohen \& Kneib (2002); Stern et al. (2010).

Using the spec- $z$ listed in Table A.1, we derive systematic shifts that we apply to the CLASH photometry as a first order template correction for Le Phare photo- $z$. These shifts are not applied to BPZ photo- $z$. Using different sets of photometry and different types of photometry change the shift's values. In Table A.2, we list the shift's values from using the different subsets of the CLASH photometry that are defined in Sect. 4.2.
Table A.1. Number of spectroscopic redshifts by cluster.

\begin{tabular}{lc}
\hline \hline Cluster & $\mathrm{Nbr}$ \\
\hline abell209 & 77 \\
abel12261 & 16 \\
abell383 & 63 \\
abel1611 & 33 \\
macs0329 & 34 \\
macs0416 & 8 \\
macs0647 & 13 \\
macs0744 & 8 \\
macs1115 & 111 \\
macs1206 & 147 \\
macs1931 & 17 \\
macs2129 & 36 \\
rxj1347 & 76 \\
rxj1532 & 3 \\
rxj2129 & 28 \\
\hline
\end{tabular}

Table A.2. Systematic shifts for sub-sets of the CLASH filters as defined in Sect. 4.2.

\begin{tabular}{lrrrrrrr}
\hline \hline Band & ACS & ACS+NIR & UVIS+ACS & UVIS+ACS+NIR & UVIS1+ACS+NIR & UVIS2+ACS+NIR & Simul4 \\
\hline$F 225 W$ & - & $0.14 \pm 0.46$ & - & $0.32 \pm 0.57$ & - & $-0.11 \pm 0.09$ \\
$F 275 W$ & - & $0.28 \pm 0.35$ & - & $0.43 \pm 0.45$ & - & $-0.05 \pm 0.07$ \\
$F 336 W$ & - & $0.05 \pm 0.23$ & - & $0.17 \pm 0.26$ & - & $0.18 \pm 0.27$ & $0.00 \pm 0.06$ \\
$F 390 W$ & - & $0.00 \pm 0.11$ & - & $0.10 \pm 0.12$ & $0.09 \pm 0.12$ & $0.10 \pm 0.12$ & $0.03 \pm 0.05$ \\
$F 435 W$ & $0.01 \pm 0.06$ & $-0.03 \pm 0.07$ & $0.05 \pm 0.06$ & $0.03 \pm 0.07$ & $0.03 \pm 0.07$ & $0.04 \pm 0.07$ & $0.03 \pm 0.05$ \\
$F 555 W$ & $0.03 \pm 0.04$ & $-0.01 \pm 0.04$ & $0.06 \pm 0.04$ & $0.03 \pm 0.05$ & $0.03 \pm 0.04$ & $0.03 \pm 0.04$ & $0.03 \pm 0.04$ \\
$F 475 W$ & $0.07 \pm 0.04$ & $0.02 \pm 0.04$ & $0.11 \pm 0.04$ & $0.08 \pm 0.06$ & $0.09 \pm 0.05$ & $0.08 \pm 0.05$ & $0.03 \pm 0.03$ \\
$F 606 W$ & $0.02 \pm 0.03$ & $0.00 \pm 0.03$ & $0.02 \pm 0.03$ & $0.00 \pm 0.03$ & $0.01 \pm 0.03$ & $0.01 \pm 0.03$ & $0.02 \pm 0.04$ \\
$F 625 W$ & $-0.01 \pm 0.02$ & $-0.03 \pm 0.02$ & $-0.01 \pm 0.02$ & $-0.04 \pm 0.02$ & $-0.02 \pm 0.02$ & $-0.03 \pm 0.02$ & $0.02 \pm 0.04$ \\
$F 775 W$ & $-0.03 \pm 0.02$ & $-0.01 \pm 0.02$ & $-0.05 \pm 0.02$ & $-0.07 \pm 0.02$ & $-0.05 \pm 0.02$ & $-0.06 \pm 0.02$ & $0.03 \pm 0.04$ \\
$F 814 W$ & $0.00 \pm 0.01$ & $0.04 \pm 0.02$ & $-0.02 \pm 0.02$ & $-0.03 \pm 0.02$ & $-0.02 \pm 0.02$ & $-0.02 \pm 0.02$ & $0.03 \pm 0.04$ \\
$F 850 L P$ & $-0.04 \pm 0.02$ & $0.03 \pm 0.02$ & $-0.07 \pm 0.02$ & $-0.08 \pm 0.02$ & $-0.06 \pm 0.03$ & $-0.07 \pm 0.02$ & $0.00 \pm 0.04$ \\
$F 105 W$ & $0.07 \pm 0.03$ & - & $0.03 \pm 0.02$ & $0.05 \pm 0.02$ & $0.03 \pm 0.02$ & $0.03 \pm 0.02$ & $-0.02 \pm 0.03$ \\
$F 110 W$ & - & - & $0.00 \pm 0.02$ & $0.03 \pm 0.02$ & $-0.00 \pm 0.02$ & $0.01 \pm 0.02$ & $-0.00 \pm 0.04$ \\
$F 125 W$ & - & - & $0.06 \pm 0.02$ & $0.09 \pm 0.02$ & $0.06 \pm 0.02$ & $0.07 \pm 0.02$ & $-0.05 \pm 0.04$ \\
$F 140 W$ & - & - & $0.06 \pm 0.03$ & $0.10 \pm 0.03$ & $0.06 \pm 0.03$ & $0.08 \pm 0.03$ & $-0.07 \pm 0.05$ \\
$F 160 W$ & - & - & - & - & - & - \\
\hline
\end{tabular}



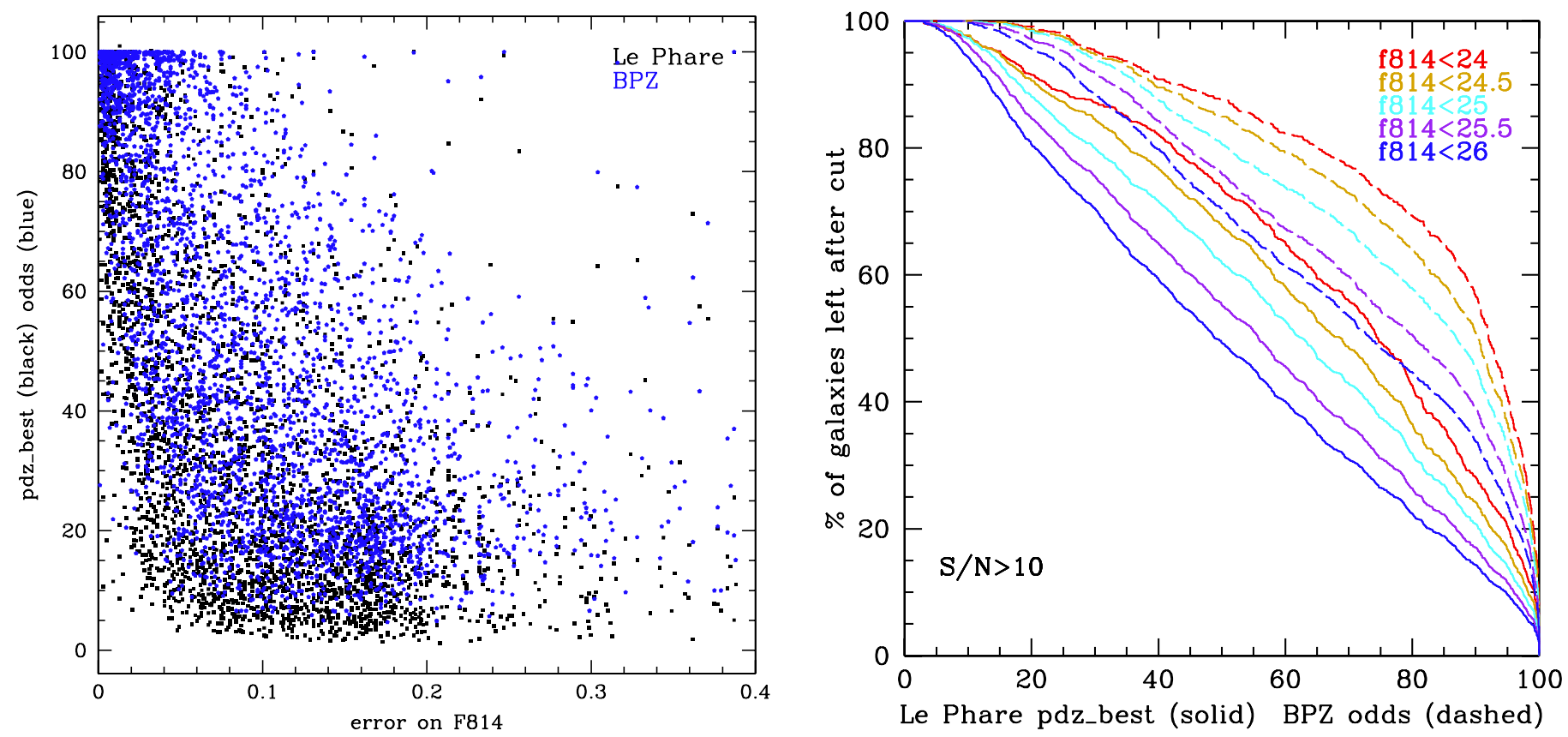

Fig. B.1. Left panel: magnitude error as a function of $o d d s$ and $p d z$ best parameter for MACS1206 galaxy cluster. The black dots and blue stars show respectively Le Phare and BPZ results. Right panel: percentage of galaxies left after a cut in the pdz_best parameter for Le Phare in solid lines and odds parameter for BPZ in dashed lines for MACS1206 galaxy cluster at different $F 814 \mathrm{~W}$ magnitudes limit.

\section{Appendix B: The odds and pdz_best photo-z confidence values}

The top panel of Fig. B.1 shows the error on the $F 814 \mathrm{~W}$ magnitude as a function of the odds parameter of BPZ in black and $p d z \_$best parameter of Le Phare in blue for MACS1206 galaxy cluster. Bright galaxies have higher odds than faint galaxies, as one can expect. We note that some Le Phare $p d z$ best values are higher than maximum possible value 100. This is a numerical issue during the integration of the $p(z)$. It usually means that the $p(z)$ is very narrow and can be assumed as high value of pdz_best.

The bottom panel of Fig. B.1 shows the percentage of galaxies left after a selection cut based on $p d z$ best Le Phare in solid lines and odds BPZ in dashed lines at different $F 814 W$ magnitude based on MACS1206 galaxy cluster photo- $z$ for all galaxies in the HST field. We note that to remove the same percentage of galaxies, one needs a higher cut for the BPZ odds parameter than with Le Phare $p d z \_b e s t$.

Figure B. 2 shows a comparison between Le Phare and BPZ when selecting the best $85 \% \mathrm{BPZ} \chi^{2}$ or Le Phare pdz_best as well as a number of detections selection.

We note that BPZ odds have values between 0 and 1 that we rescale at 0 to 100 in order to have the same scale between Le Phare $p d z \_$best and BPZ odds.

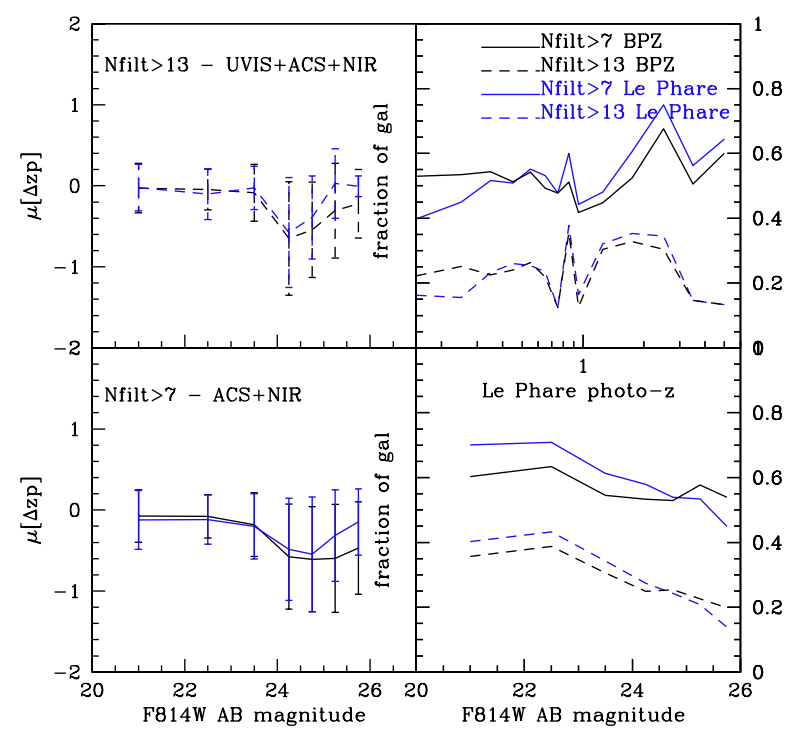

Fig. B.2. Le Phare and BPZ photo- $z$ comparison for samples of galaxies selected as a function of the number of detections using the best $85 \%$ $\mathrm{BPZ} \chi^{2}$ or Le Phare $p d z$ best values. The left panels show the mean values of BPZ-Le Phare photo- $z$ for the different samples. The right panels show the fraction of galaxies selected by bins of magnitude and redshift. 


\section{Appendix C: Arcs}

In this section, we show the photo- $z$ results from the BPZ code for the standard isophotal photometry for each of the lensed images of MACS1206 systems. These results are to be compared with those shown in Sect. 5 for Le Phare. We show stamps images of each arcs, SExtractor segmentation map for the isophotal photometry, the probability of the photo- $z$ an the best-fit template of the BPZ results from the first left column to last column. The aperture shape of the SExtractor isophotal photometry is showed by the black contour on the second column from the left. When comparing these figures to Fig. 12 we note that the tailored photometry and the isophotal SExtractor photometry have different shapes. Photo- $z$ derive from both shapes using the same photo- $z$ code usually prefers the tailored photometry as explained in Sect. 5 . 
S. Jouvel et al.: Photo- $z$ for the CLASH survey
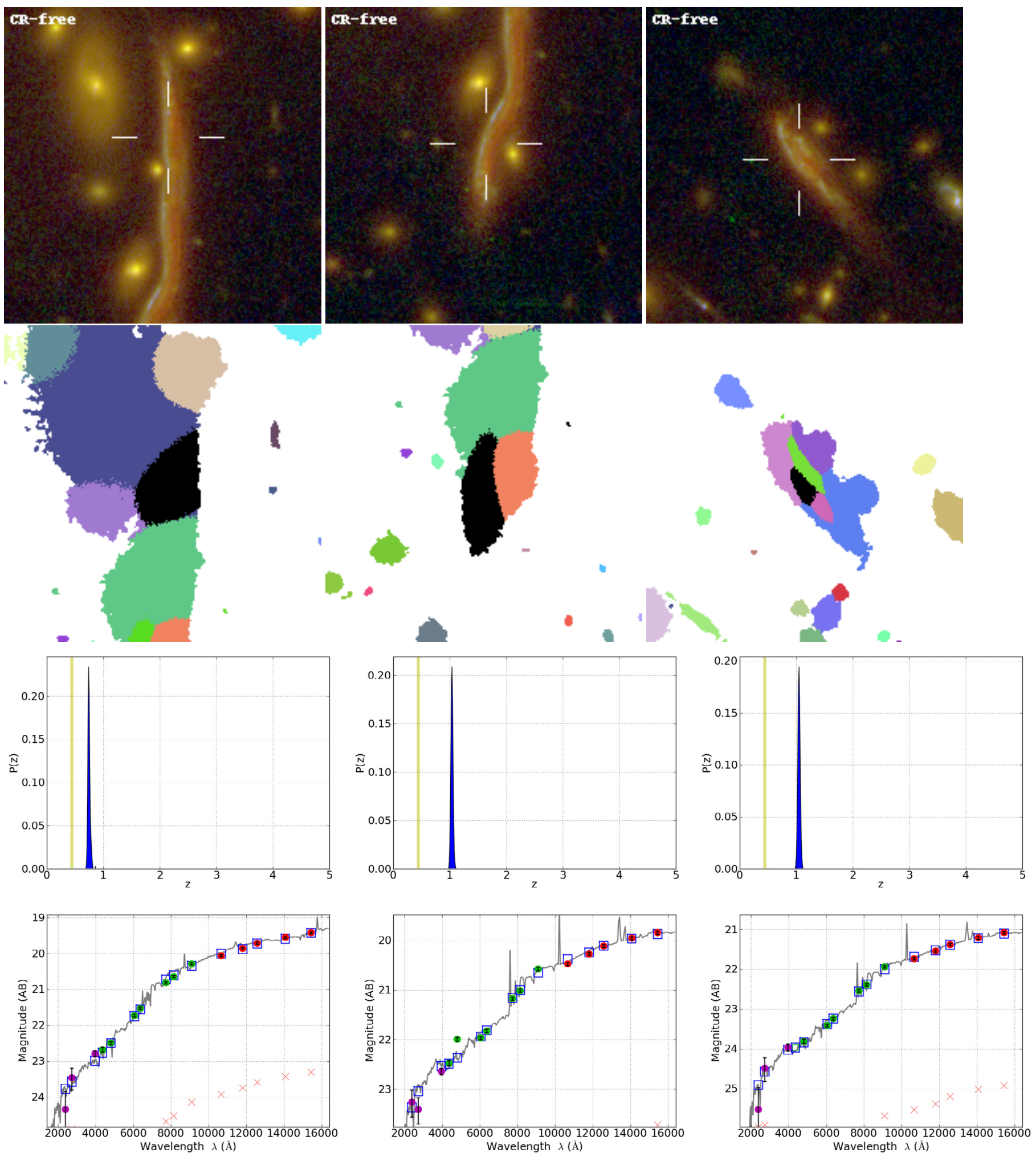

Fig. C.1. BPZ photometric redshift results for the strongly lensed system 1 standard photometry from SExtractor isophotal apertures. From top to bottom, figures are cut-out images of the arcs, segmentation map of the isophotal photometry, probability of the photometric redshift and best-fit template for the arcs considered. From left to right, figures show arc 1.1, 1.2, and 1.3. 

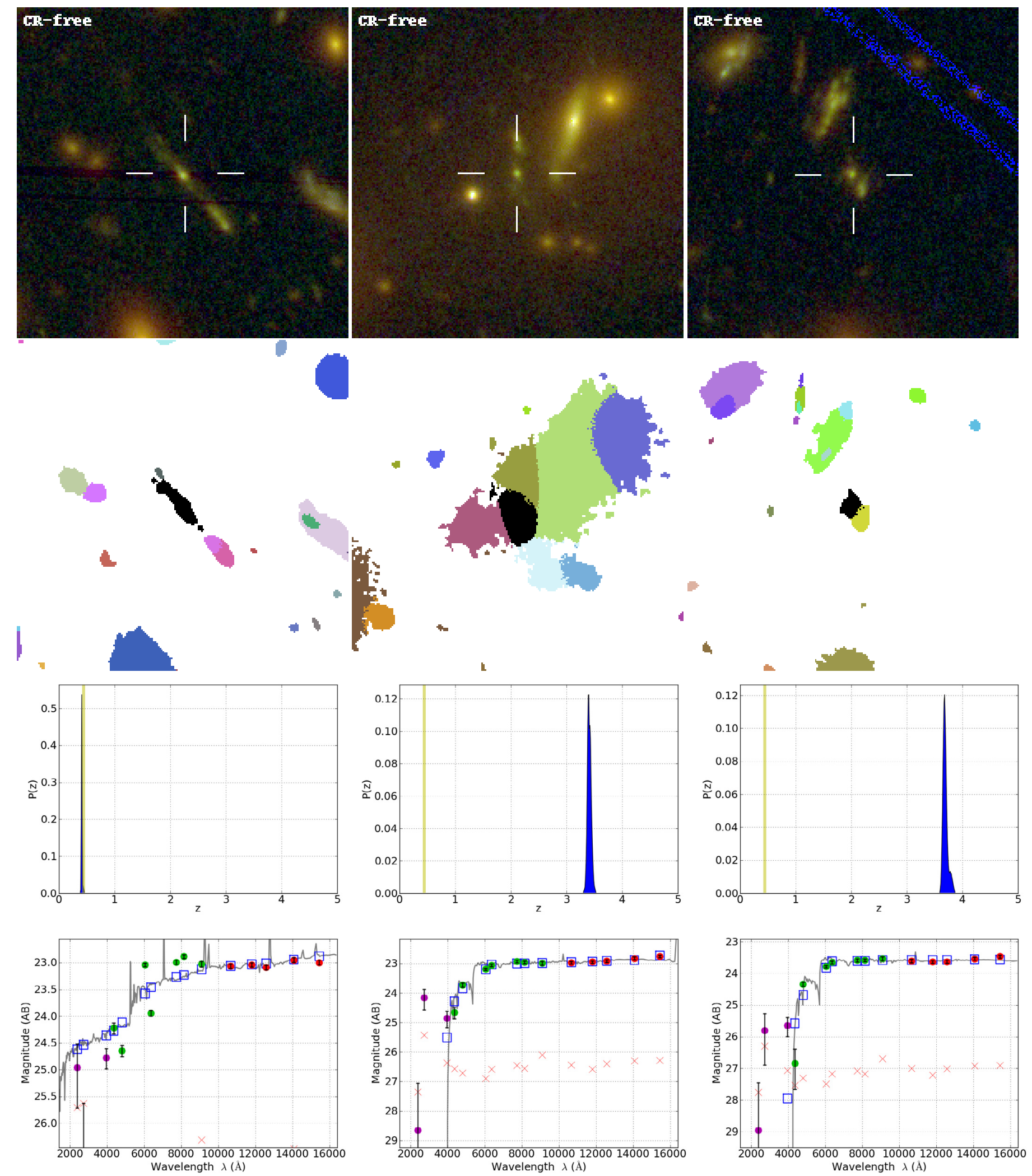

Fig. C.2. BPZ photometric redshift results for the strongly lensed system 2 standard photometry from SExtractor isophotal apertures. From top to bottom, figures are cut-out images of the arcs, segmentation map of the isophotal photometry, probability of the photometric redshift and best-fit template for the arcs considered. From left to right, figures show arc 2.1, 2.2, and 2.3. 
S. Jouvel et al.: Photo- $z$ for the CLASH survey
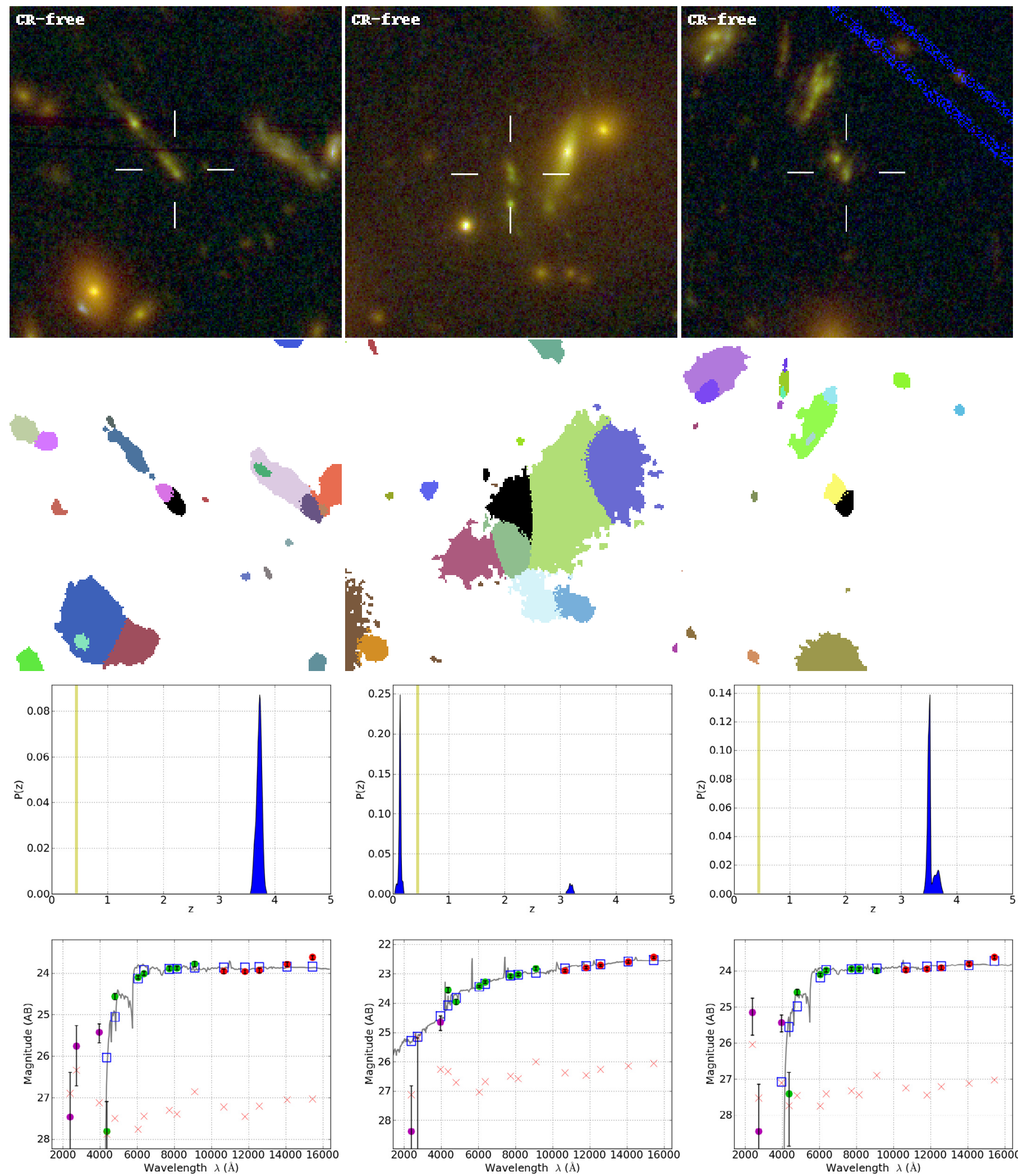

Fig. C.3. BPZ photometric redshift results for the strongly lensed system 3 standard photometry from SExtractor isophotal apertures. From top to bottom, figures are cut-out images of the arcs, segmentation map of the isophotal photometry, probability of the photometric redshift and best-fit template for the arcs considered. From left to right, figures show arc 3.1, 3.2, and 3.3. 

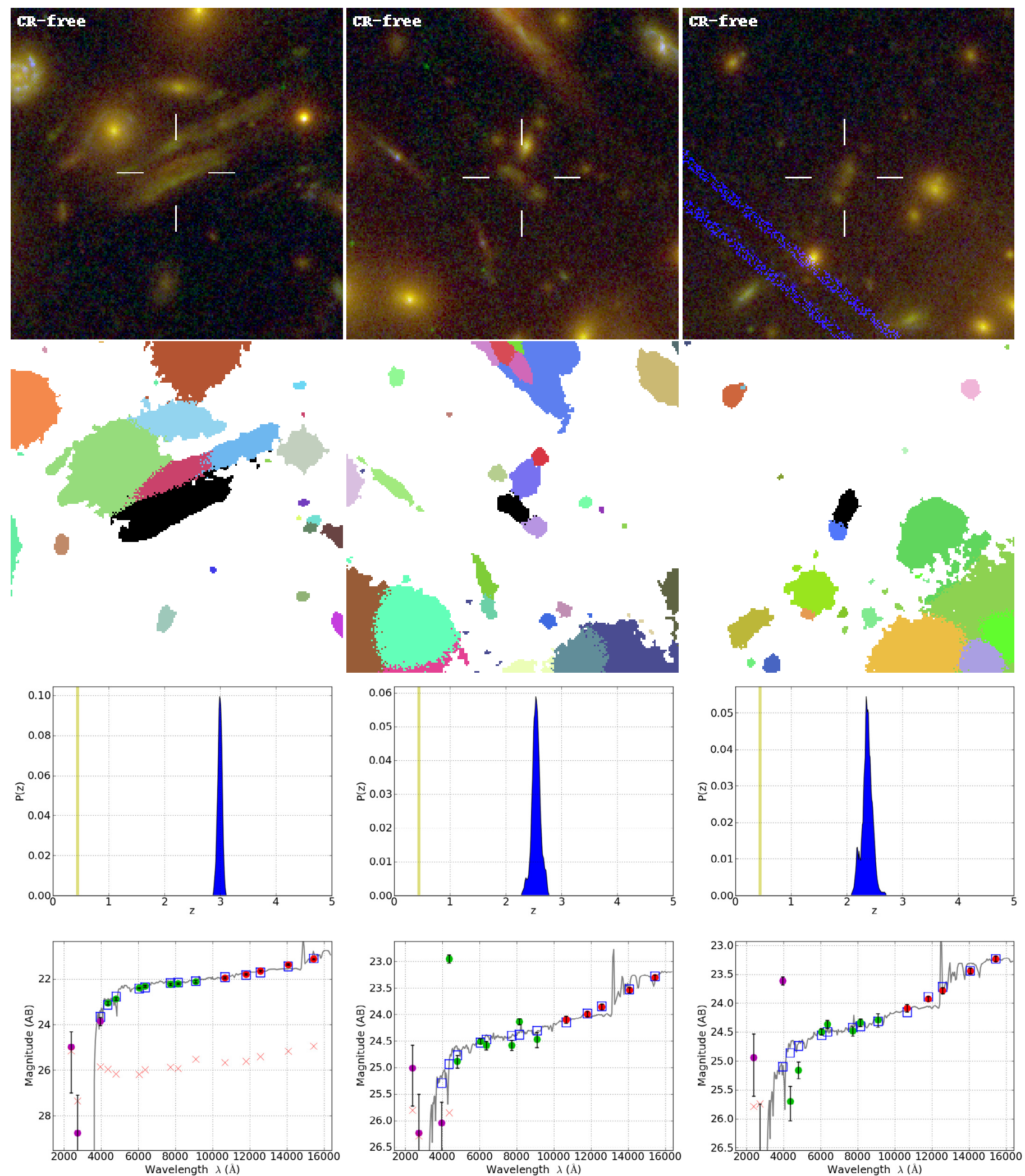

Fig. C.4. BPZ photometric redshift results for the strongly lensed system 4 standard photometry from SExtractor isophotal apertures. From top to bottom, figures are cut-out images of the arcs, segmentation map of the isophotal photometry, probability of the photometric redshift and best-fit template for the arcs considered. From left to right, figures show arc 4.1, 4.2, 4.3. 
S. Jouvel et al.: Photo- $z$ for the CLASH survey

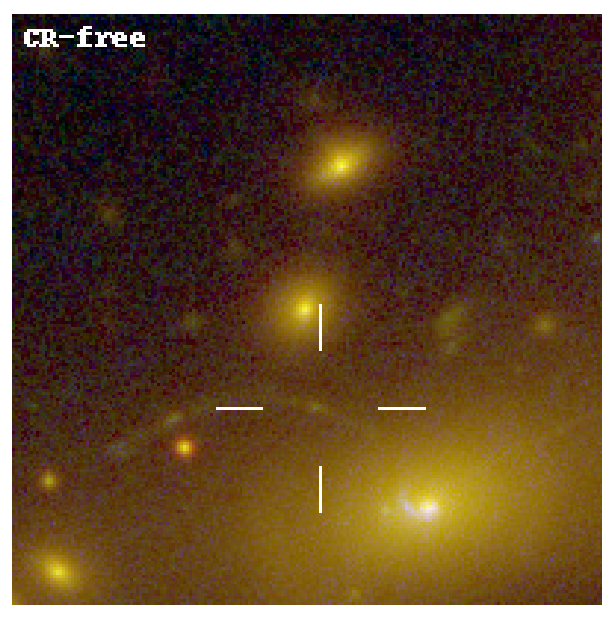

\section{CR-free}
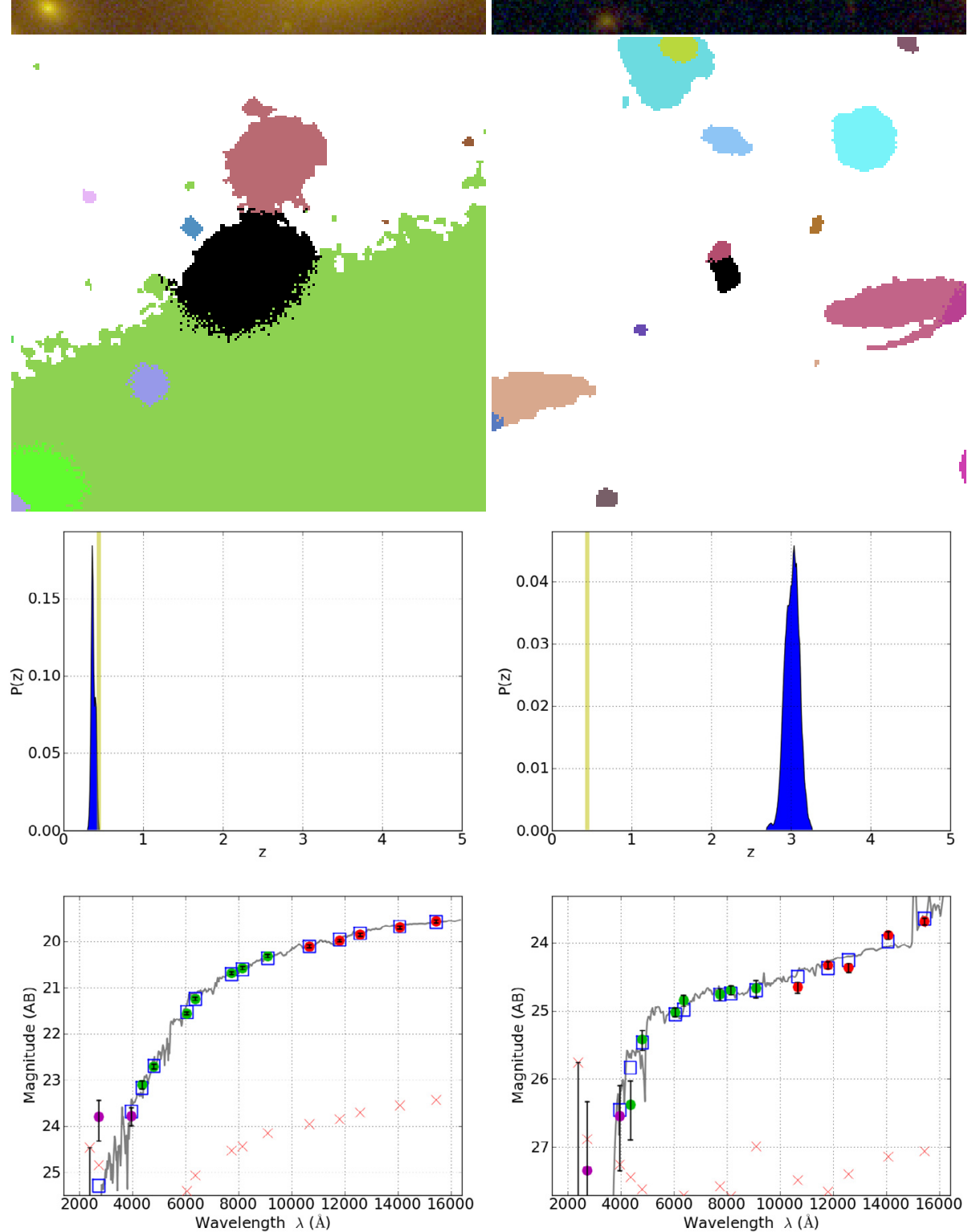

Fig. C.5. BPZ photometric redshift results for the strongly lensed system 4 standard photometry from SExtractor isophotal apertures. From top to bottom, figures are cut-out images of the arcs, segmentation map of the isophotal photometry, probability of the photometric redshift and best-fit template for the arcs considered. From left to right, figures show arc 4.4 and 4.5. 